\title{
Cognitive-Behavioral Psychotherapy for Anxiety and Depressive Disorders in Children and Adolescents: An Evidence-Based Medicine Review
}

\author{
SCOTT N. COMPTON, Ph.D., JOHN S. MARCH, M.D., M.P.H., DAVID BRENT, M.D., \\ ANNE MARIE ALBANO, Ph.D., V. ROBIN WEERSING, Ph.D., AND JOHN CURRY, Ph.D.
}

\begin{abstract}
Objective: To review the literature on the cognitive-behavioral treatment of children and adolescents with anxiety and depressive disorders within the conceptual framework of evidence-based medicine. Method: The psychiatric and psychological literature was systematically searched for controlled trials applying cognitive-behavioral treatment to pediatric anxiety and depressive disorders. Results: For both anxiety and depression, substantial evidence supports the efficacy of problem-specific cognitive-behavioral interventions. Comparisons with wait-list, inactive control, and active control conditions suggest medium to large effects for symptom reduction in primary outcome domains. Conclusions: From an evidence-based perspective, cognitive-behavioral therapy is currently the treatment of choice for anxiety and depressive disorders in children and adolescents. Future research in this area will need to focus on comparing cognitivebehavioral psychotherapy with other treatments, component analyses, and the application of exportable protocol-driven treatments to divergent settings and patient populations. J. Am. Acad. Child Adolesc. Psychiatry, 2004;43(8):930-959.

Key Words: outcome studies, children and adolescents with major depression and dysthymic disorder, children and adolescents with anxiety disorder, literature review.
\end{abstract}

Due in part to a productive interplay between research and clinical practice (Rutter, 1999), many clinical researchers now believe that cognitive-behavioral therapy (CBT) administered within an evidence-based, multimodal, multidisciplinary practice model is the psychotherapeutic treatment of choice for youth with internalizing disorders (Geddes et al., 1997; March and Wells, 2003). In this context, the past 10 years witnessed the emergence of diverse, sophisticated, and empirically supported CBTs covering the range of childhood-onset anxiety and depressive disorders

Accepted November 17, 2003.

Drs. Compton, March, and Curry are with the Department of Psychiatry and Behavioral Psychology, Duke University Medical Center, Durham, NC; Dr. Brent is with Western Psychiatric Institute and Clinic, Pitsburgh, PA; Dr. Albano is with New York University School of Medicine, NY; and Dr. Weersing is with the Yale Child Study Center, New Haven, CT.

Correspondence to Dr. Compton, Duke University Medical Center, Box 3527, Durham, NC 27710; e-mail: scompton@duke.edu.

0890-8567/04/4308-0930@2004 by the American Academy of Child and Adolescent Psychiatry.

DOI: 10.1097/01.chi.0000127589.57468.bf
(Bernstein and Shaw, 1997; Birmaher et al., 1996a,b). Using the tools of evidence-based medicine (EBM) (Sackett et al., 1997), this article provides a critical review of CBT for these conditions. We do not address obsessive-compulsive disorder and posttraumatic stress disorder, for which recent critical reviews are available (Cohen et al., 2000; Franklin et al., 2002; March, 1995), or bipolar disorder, for which cognitivebehavioral interventions are just now emerging (McClellan and Werry, 1997). The reader interested in a "how-to-do-it" perspective may wish to pursue recent overviews of CBT (Hibbs and Jensen, 1996; Reinecke et al., 2003) interventions for childhood-onset anxiety (Kendall et al., 1999, 2000, 2003; March and Mulle, 1998; Rapee et al., 2000; Silverman and Kurtines, 1996) and depressive disorders (Brent et al., 1997; Clarke et al., 1990).

GUIDING THEORY

Although a comprehensive review of the theoretical rationale of CBT is clearly beyond the scope of this article (for a still cogent précis, see Kendall, 1993; Ken- 
dall and Panichelli-Mindel, 1995), a short overview is heuristically valuable. Historically, behavior therapy (the BT in CBT) evolved within the theoretical framework of classical and operant conditioning, with cognitive interventions (the $\mathrm{C}$ in $\mathrm{CBT}$ ) assuming a more prominent role with the increasing recognition that person-environment interactions are mediated by cognitive processes (Van Hasselt and Hersen, 1993). Looked at in the context of situational and/or cognitive processes, BT is sometimes referred to as nonmediational (emphasizing the direct influence of situations on behavior) and CT as mediational (emphasizing that thoughts and feelings underlie behavior). Hence, behavioral psychotherapists work with patients to change behaviors and thereby to reduce distressing thoughts and feelings. Cognitive therapists work to first change thoughts and feelings, with improvements in functional behavior following in turn.

Although CBT is often referred to as a unitary treatment, it is actually a diverse collection of complex and subtle interventions that must each be mastered and understood from the social learning perspective. Subsequently, a cognitive-behavioral case formulation guides the therapist in administering treatment techniques in a flexible manner for the patient presenting with any one disorder or comorbid presentation of mental disorders (for an overview of a modular approach to CBT interventions, see Curry and Reinecke [2003]). Nonetheless, despite their seeming differences, cognitive-behavioral interventions typically share five qualities: (1) adherence to the scientist-clinician model, whereby treatments are chosen based on demonstrated evidence or are applied within a case evaluation format to determine efficacy; (2) a thorough idiographic assessment (e.g., functional analysis) of target behaviors and the situational, cognitive, and behavioral factors that have established or are maintaining the symptoms of interest (for a detailed overview of how to conduct a functional analysis, see Haynes and O'Brien [1990]); (3) an emphasis on psychoeducation; (4) problem-specific treatment interventions designed to ameliorate the symptoms of concern; and (5) relapse prevention and generalization training at the end of treatment. For example, using cognitive restructuring and exposure-based interventions, CBT for anxiety disorders encourages cognitions and behaviors designed to promote habituation or extinction of inappropriate fears. Likewise, CBT for depression directly confronts maladaptive depressogenic cognitions, including helplessness, hopelessness, and hostility, and aims behaviorally to reconstitute pleasant relationships, be they intrapsychic, interpersonal, school, or spiritual. As evidence-based therapies, each is supported by a more or less robust research literature, and manuals are usually available to guide practitioners in using CBT for specific problems. Thus, CBT fits nicely into the current medical practice environment that appropriately values empirically supported, brief, problem-focused treatments.

From this vantage point, CBT represents a developmentally sound approach to pediatric mental illness. Children normally acquire social-emotional (self and interpersonal) competencies across time. The failure to do so, relative to age, gender, and culture-matched peers, may reflect capacity limitations, individual differences in the rate of skill acquisition for specific competencies, environmental factors, and/or the development of a mental illness. In CBT, the task of the mental health practitioner is to understand the presenting symptoms in the context of child-specific constraints to normal development and to devise a tailored treatment program that eliminates those constraints so that the youngster can resume a normal developmental trajectory insofar as is possible.

To the extent that symptom relief occurs, it can be assumed that improvement reflects concurrent changes (e.g., learning) in the CNS (Andreason, 1997; Hyman, 2000). Thus, the cognitive-behavioral treatment of pediatric mental illness can be thought of as partially analogous to the treatment of, for example, juvenileonset diabetes, with the caveat that the target organ, the brain in the case of major mental illness, requires interventions of much greater complexity. Although medications are of importance-in diabetes, insulin, and in the anxiety or affective disorders, a serotonin reuptake inhibitor-the critical point is that each also involves crucial psychosocial interventions that work in part by biasing the somatic substrate of the disorder toward more normal functioning (Hyman, 2000). In diabetes, the behavioral intervention of choice is diet and exercise, and in the anxiety or affective disorders, it is cognitive-behavioral psychotherapy.

\section{METHOD}

EBM has emerged as a promising paradigm for medical practice (for a comprehensive review, see Sackett et al. [2000]) and is clini- 
cally akin to the scientist-practitioner model in academic psychology (Barlow, 1993). EBM deemphasizes the more typical reliance on unsystematic clinical experience as a sufficient ground for clinical decision making. Instead, EBM stresses the examination of evidence from systematic diagnostic assessment technologies and clinical research as a tool to inform clinical practice, and it provides a heuristically valuable organizing focus for the individual clinician seeking to transition efficacy and effectiveness studies into clinical practice at the level of the individual patient (Geddes et al., 1997).

Using established EBM criteria for assessing the validity of treatment studies as guides to clinical practice (Guyatt et al., 1993, 1994, 1999), a search for relevant literature was conducted via Medline and PsycINFO, using the following text terms: anxiety, depression, cognitive therapy, and behavior therapy. Only randomized, controlled trials (RCTs) for individuals with a specific disorder were included. Additionally, to be included, articles must have met the following criteria: published in an English-language, peerreviewed journal between 1990 and 2002; included children between the ages of 8 and 18; included an outcome measure of known clinical significance; and used an analytic strategy consistent with the study design. A follow-up assessment was preferred but not required. Excluded from consideration were articles concerning the treatment of obsessive-compulsive disorder, posttraumatic stress disorder, or bipolar disorder; included were articles concerning the treatment of specific phobias, social phobia, selective mutism, overanxious disorder, separation anxiety disorder, panic disorder, generalized anxiety disorder, major depression, and dysthymia.

The text of this article is supported by a series of tables that summarize the main findings of each study identified during the literature search. The tables are organized by type of disorder (anxiety versus depression); within each disorder, separate tables summarize findings at post-treatment and at long-term follow-up.

The information presented in each table includes study citation (studies are listed in alphabetical order by first author), research design (type, control condition, analysis sample), sample information (total number, age range, percentage of males, and ethnicity), the diagnoses targeted by the intervention, brief details about the intervention, primary dependent measures (both categorical and scalar), sample size in each treatment condition, proportion of sample responding, magnitude of the treatment effect (portrayed in terms of number needed to treat $[\mathrm{NNT}]$ and standardized effect size estimates), and general comments by the authors.

The NNT is a measure of the average response, presented as the probability of response in single patient units. Arithmetically, the NNT is the inverse of the absolute risk reduction (1/ARR), defined as the percentage of response in the experimental group minus the percentage of response in the control condition. In practice, NNT represents the number of patients who need to be treated with the active treatment to produce one additional positive outcome beyond that obtainable with the control or comparison condition. For example, an NNT of 10 describes the number of patients whom a clinician would need to treat with the active treatment rather than the control treatment to see one additional positive outcome. A very small NNT (that is, an NNT that approaches 1) suggests that a favorable outcome occurs in nearly every patient who receives the treatment and in relatively few patients in the comparison group. An NNT of 2 or 3 indicates that a treatment is quite effective.

Standardized effect size estimates were calculated with the assistance of ES (Shadish et al., 1999), a computer software program designed to calculate effect size estimates from published studies. ES calculates the standardized mean difference statistic, commonly referred to as Cohen's $d$ and computed as $d=\left(M_{t}-M_{c}\right) / \mathrm{SD}$, where
$M_{t}$ is the mean of the treatment group, $M_{c}$ is the mean of the comparison group, and SD is the pooled within-group standard deviation. All effect size estimates are reported such that positive scores indicate that the treatment group improved more than the comparison group.

\section{TREATMENT OF ANXIETY DISORDERS}

To their advantage, cognitive-behavioral therapists have a robust literature validating the effectiveness of specific psychological techniques for anxiety disorders and a steadily growing literature supporting the use of prescriptive treatment protocols for these disorders.

\section{Types of Investigations}

Twenty-one RCTs evaluating a variety of cognitivebehavioral interventions for the treatment of child and adolescent anxiety disorders were identified (Table 1). As a group, these studies are noteworthy for their methodological rigor and the systematic way in which they have advanced the understanding of childhood anxiety disorders and how best to treat this important population. With respect to methodological rigor, all studies used contrasting group designs in which active treatments were compared with either a wait-list or notreatment control condition (Cornwall et al., 1996; Hayward et al., 2000; Kendall, 1994; Kendall et al., 1997; King et al., 1998; Shortt et al., 2001; Silverman et al., 1999a) or an attention placebo-controlled condition (Beidel et al., 2000; Last et al., 1998; Muris et al., 2002). Moreover, several studies compared more than one active treatment condition (Barrett, 1998; Barrett et al., 1996; Beidel et al., 2000; Cobham et al., 1998; Flannery-Schroeder and Kendall, 2000; Mendlowitz et al., 1999; Menzies and Clarke, 1993; Muris et al., 2001; Nauta et al., 2003; Silverman et al., 1999b; Spence et al., 2000).

Investigators in this area have also systematically evaluated a variety of clinically relevant questions: for instance, whether group CBT is more effective than individual CBT (Flannery-Schroeder and Kendall, 2000; Manassis et al., 2002; Muris et al., 2001), whether adding parental participation enhances treatment outcomes (Barrett, 1998; Barrett et al., 1996; Cobham et al., 1998; Mendlowitz et al., 1999; Nauta et al., 2003; Shortt et al., 2001; Spence et al., 2000), whether concurrent treatment of parental anxiety enhances treatment outcomes (Cobham et al., 1998), and whether two active treatment components, which are 
RESEARCH UPDATE REVIEW: CBT

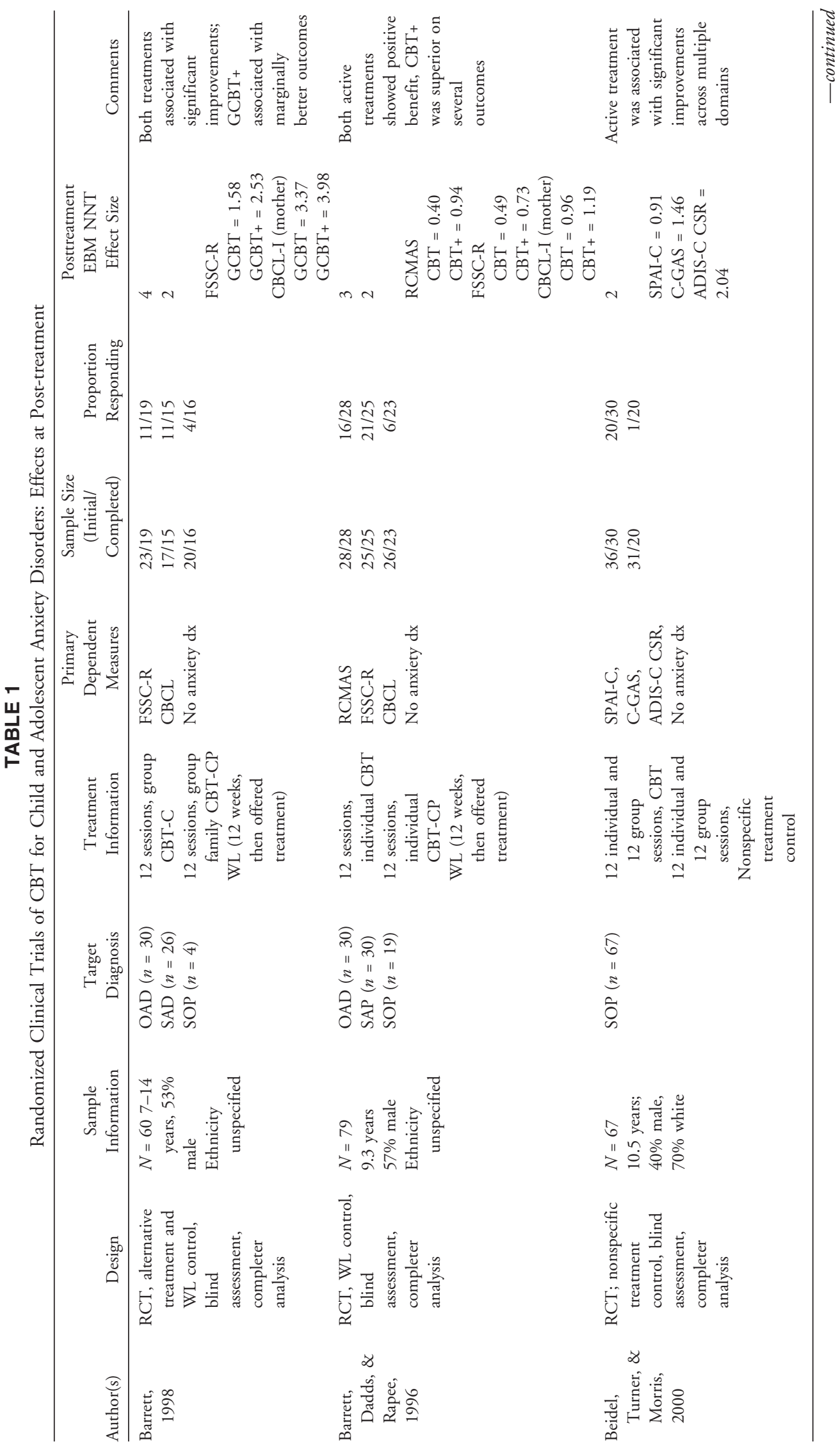




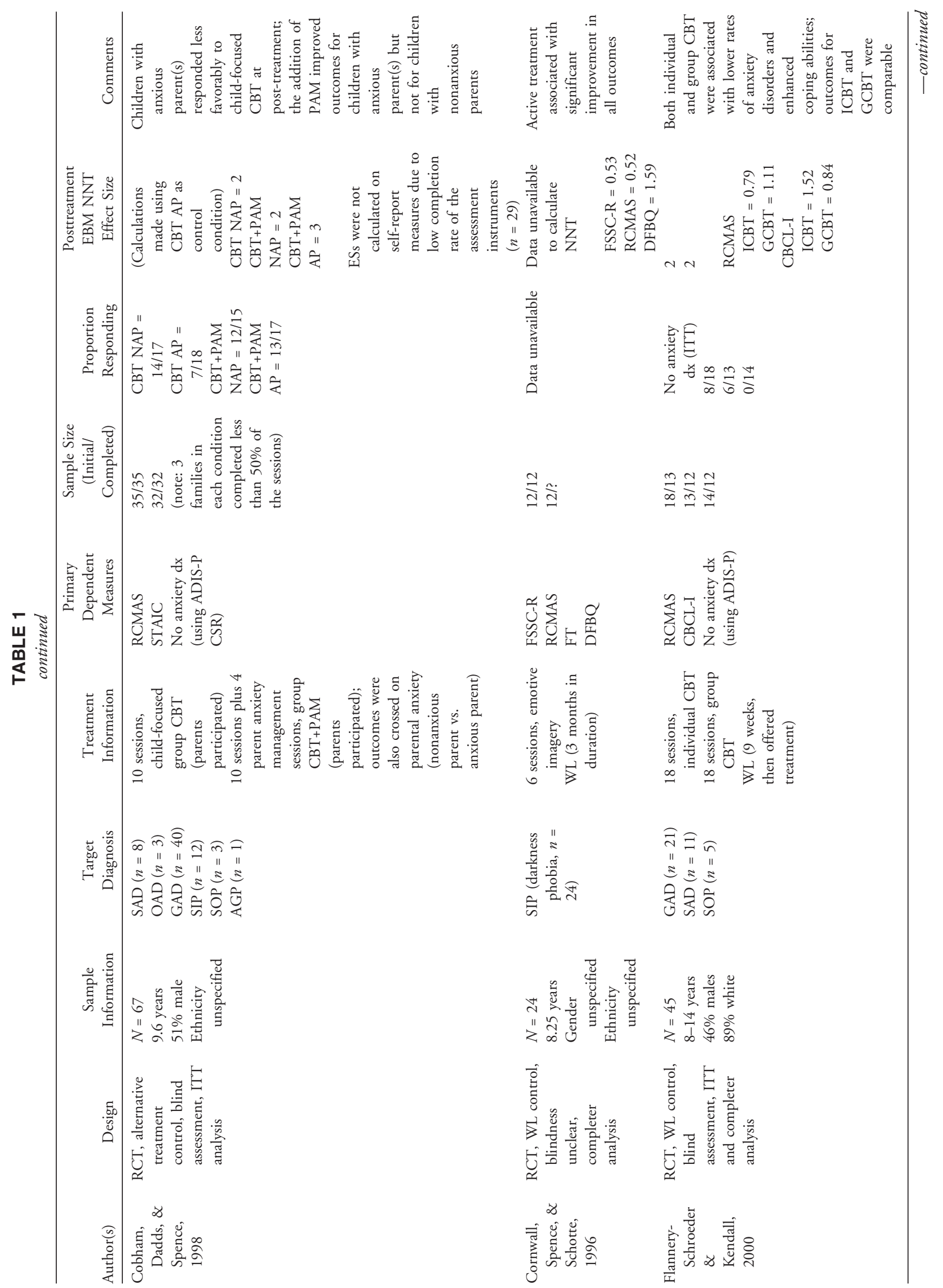




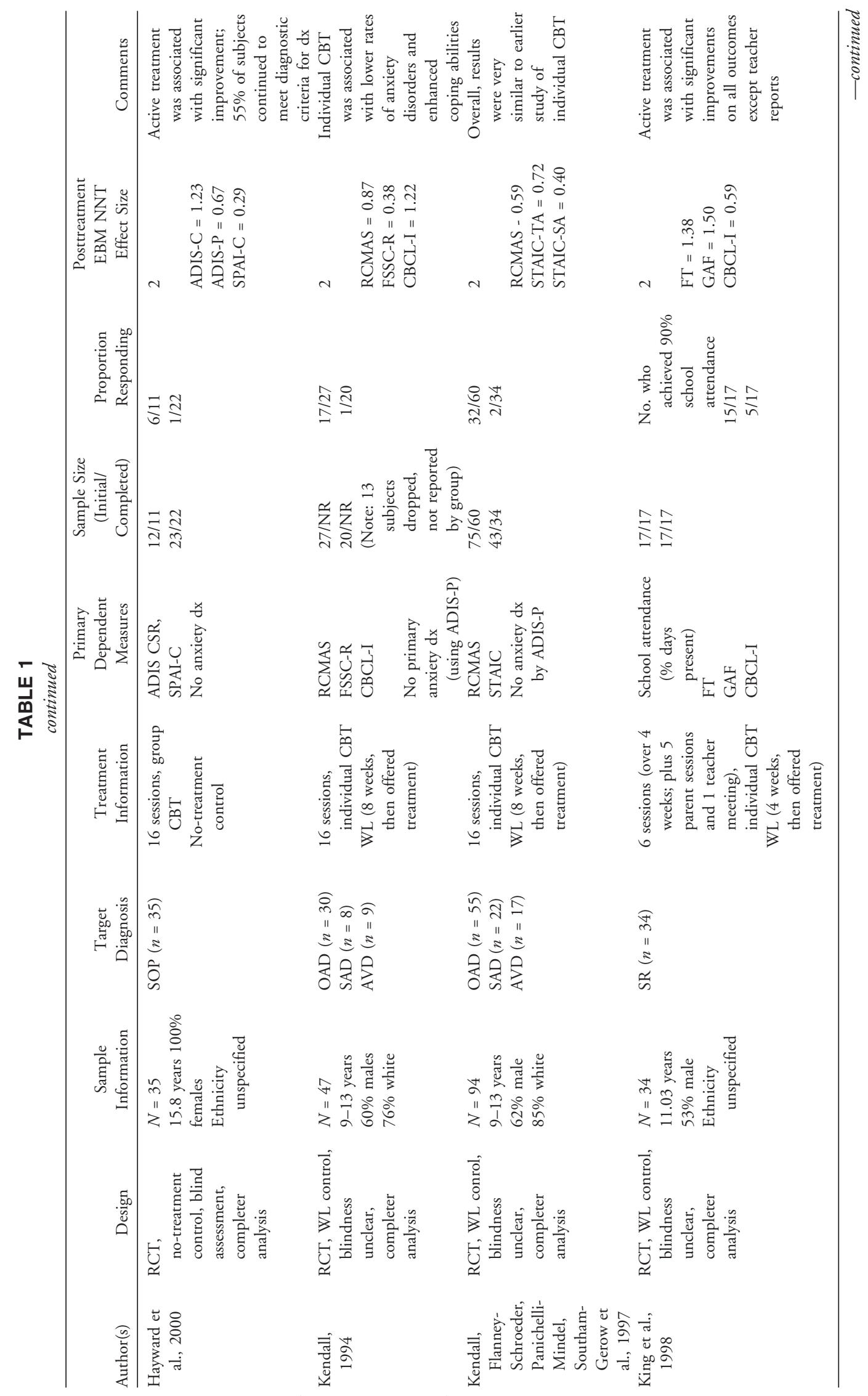




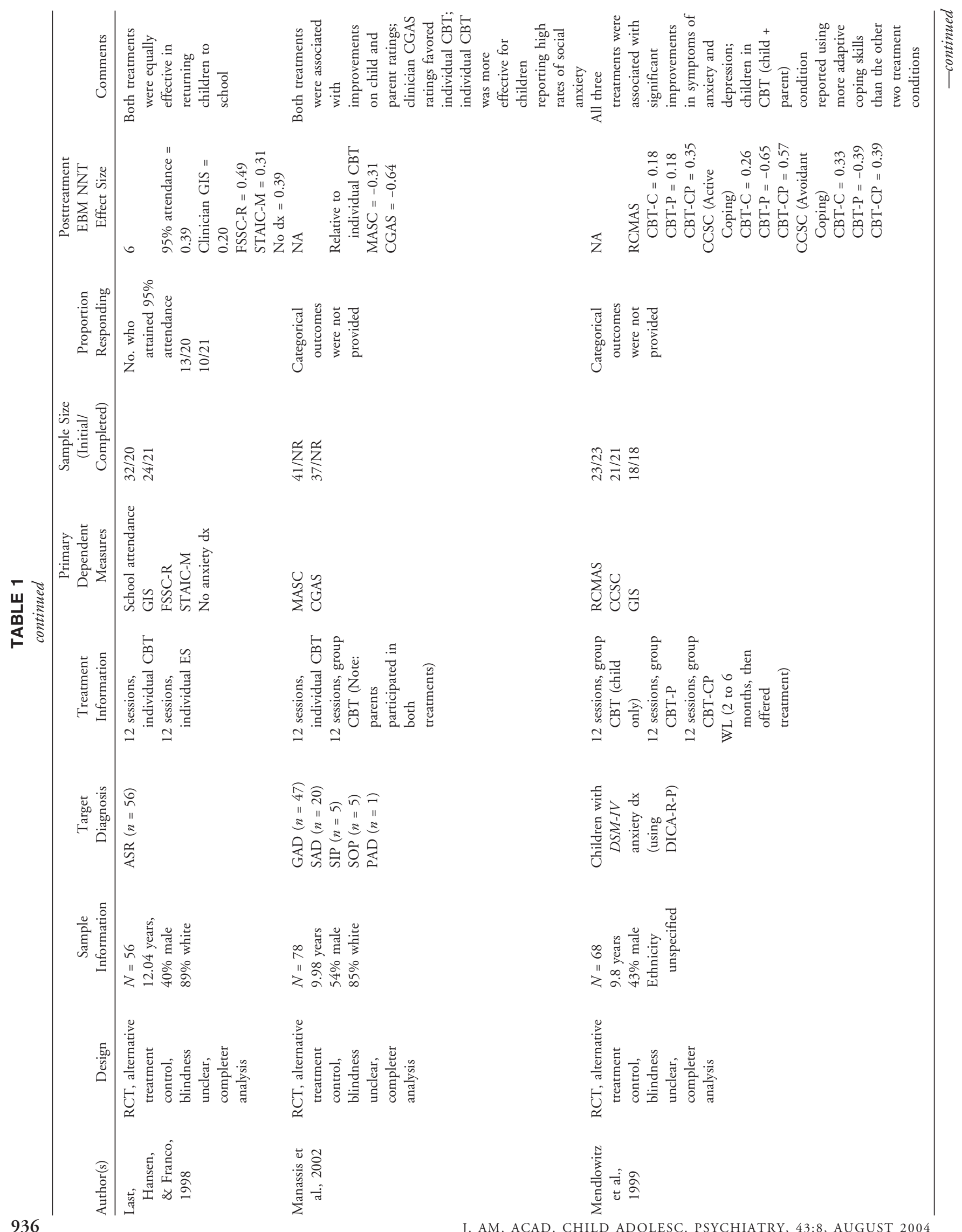


RESEARCH UPDATE REVIEW: CBT

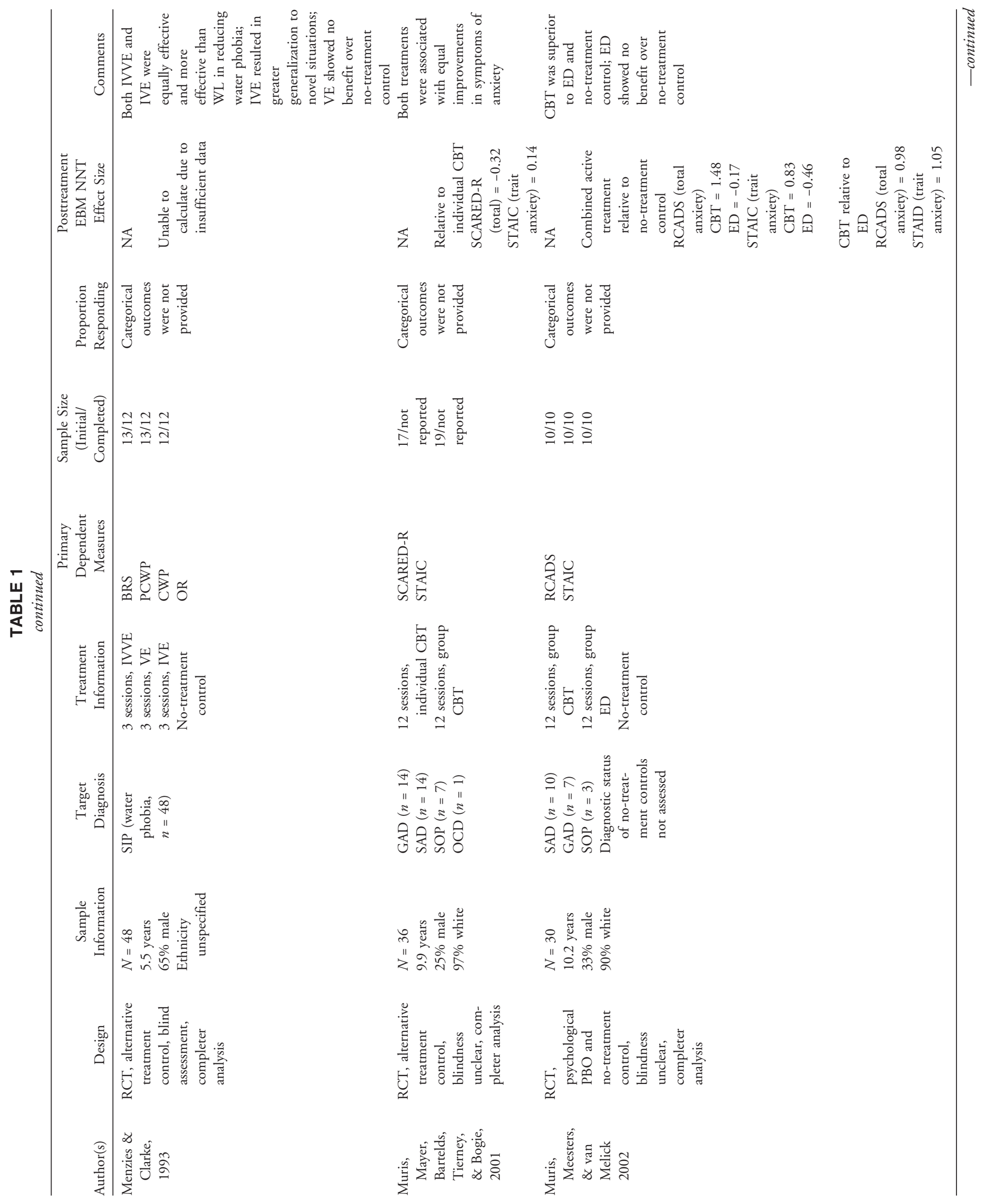

J. AM. ACAD. CHILD ADOLESC. PSYCHIATRY, 43:8, AUGUST 2004 


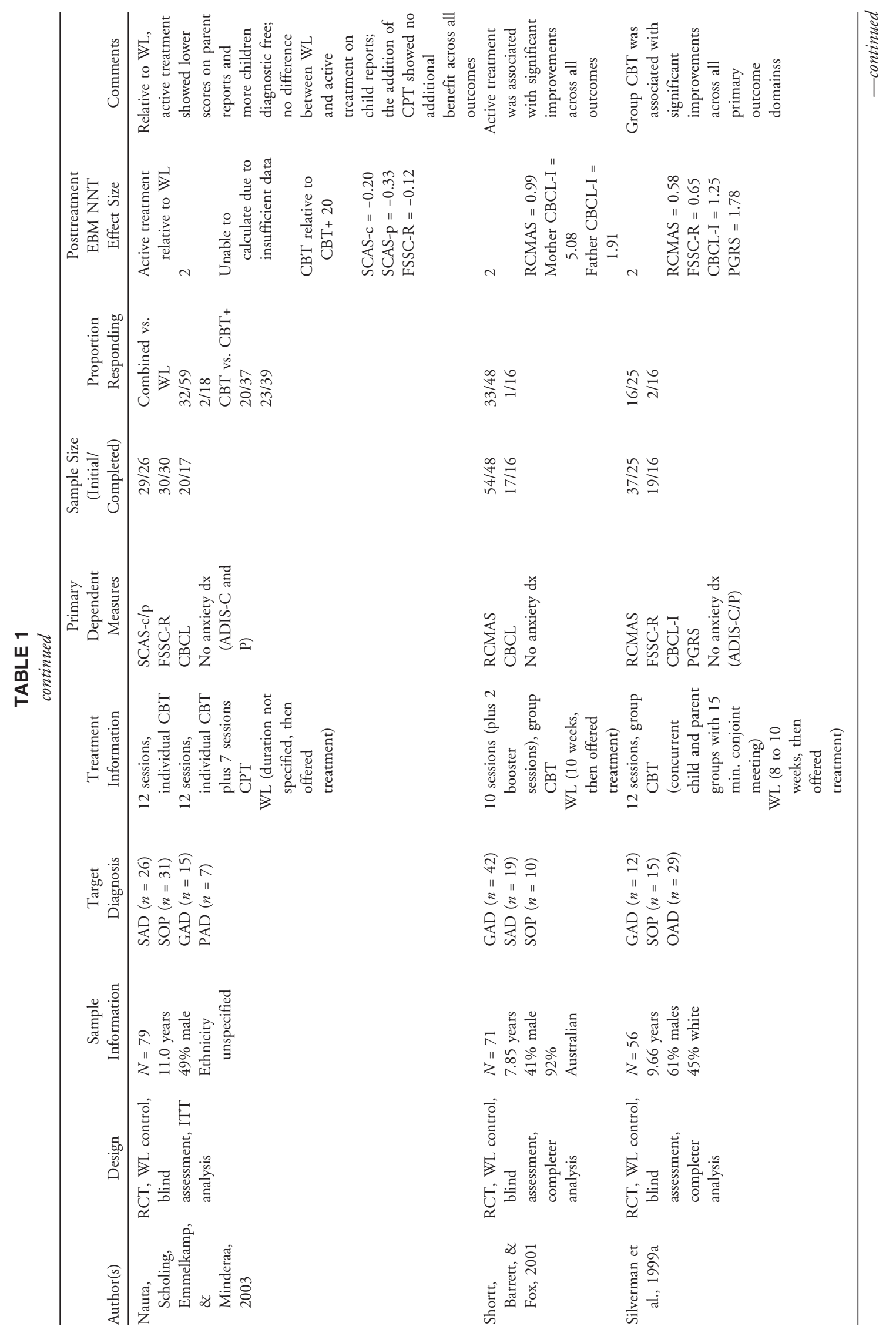




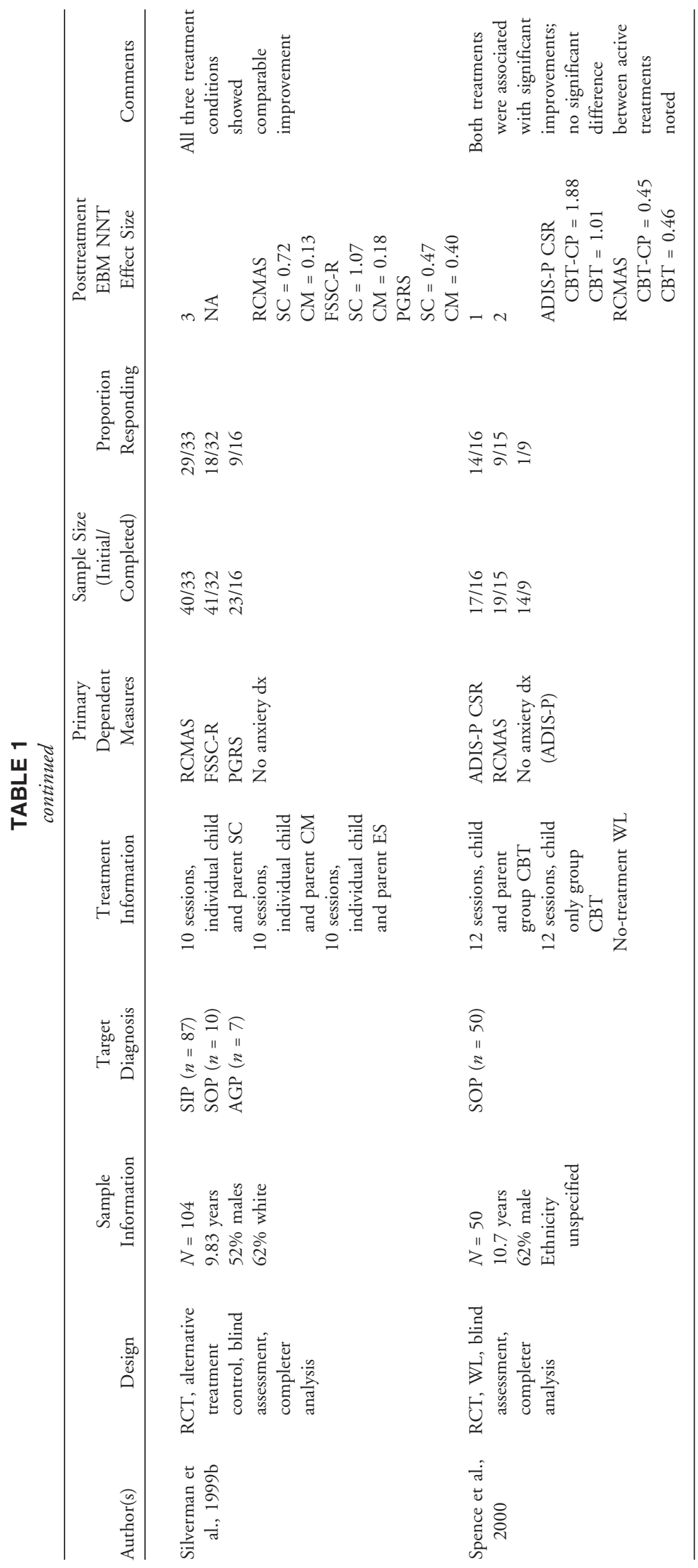

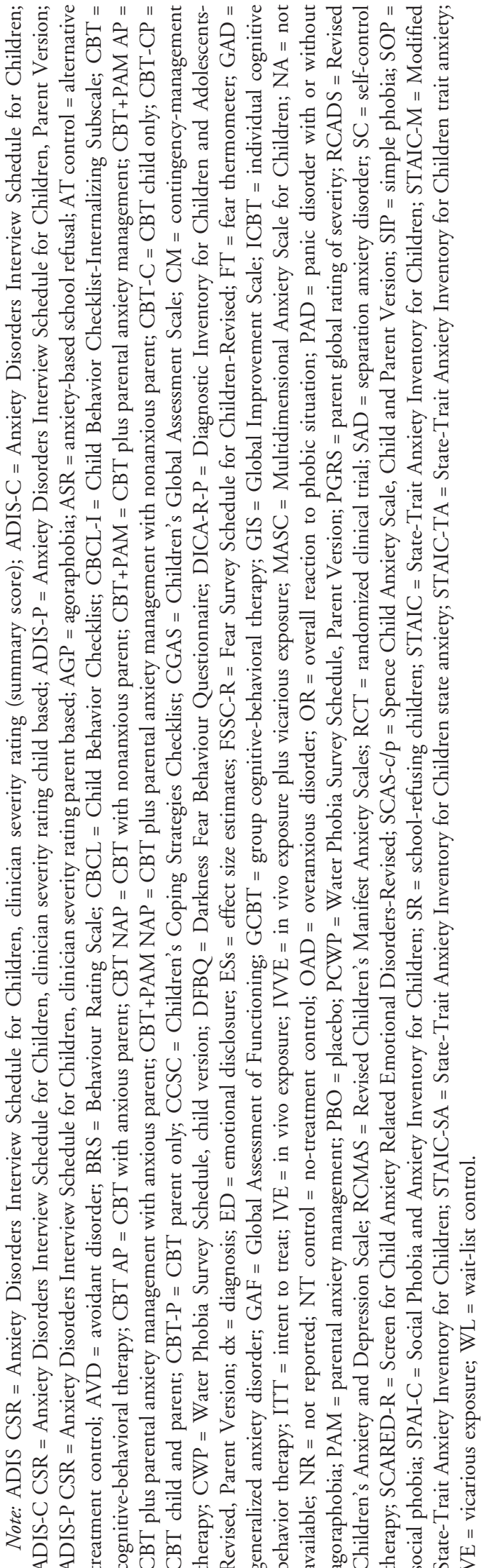


often combined in traditional cognitive-behavioral protocols (e.g., behavioral contingency management versus cognitive self-control), are differentially effective (Silverman et al., 1999b). Moreover, several of the studies cited were replications and extensions of existing protocols by independent researchers (Barrett, 1998; Barrett et al., 1996; Manassis et al., 2002; Mendlowitz et al., 1999; Muris et al., 2001, 2002).

\section{Assessment Issues}

Diagnosis and Symptom Profile. Valid and reliable assessment is essential to the skillful application and evaluation of cognitive-behavioral treatments (Thyer, 1991) and is a strength of the cited studies taken as a whole. All but 2 of the 21 studies cited in Table 1 (Cornwall et al., 1996; Menzies and Clarke, 1993) used semistructured clinical interviews to identify subjects as having an anxiety disorder as well as documenting diagnostic comorbidities and assessing treatment outcomes. By a significant margin (13 of 21), the most widely used semistructured clinical interview was the Anxiety Disorders Interview Schedule, Child and Parents Versions (ADIS-C/P) (Silverman, 1987; Silverman and Albano, 1996a,b; Silverman and Nelles, 1988). This interview is most commonly administered separately to children and parents, and then data are combined from both sources to derive a final "composite" diagnosis; however, several studies deviated from this standard practice and relied solely on information obtained from parents to determine diagnostic status (Shortt et al., 2001; Spence et al., 2000) and treatment outcome (Cobham et al., 1998; FlannerySchroeder and Kendall, 2000; Kendall, 1994; Kendall et al., 1997; Shortt et al., 2001; Spence et al., 2000). In addition to providing a diagnosis, the ADIS requires the clinician to provide a clinician severity rating (CSR). The CSR is the clinician's estimate of the degree of functional impairment and distress engendered by the disorder (Albano and Silverman, 1996). Unfortunately, only two studies characterized the sample in terms of the CSR (Hayward et al., 2000; Silverman et al., 1999a). Because the CSR may predict the nature and outcome of treatment, the failure of researchers to adequately characterize the baseline characteristics of their sample along this dimension is a notable deficiency.

Demographics and Severity. Both genders are largely represented in the treated population, with only one study containing a sample that was limited to females (Hayward et al., 2000). Although the majority of studies attempted to recruit children and adolescents, the average age of subjects across all studies was approximately 9.85 years. This leaves open the question of generalizability of the research findings, as well as protocol-driven interventions, to older adolescent populations. Other demographic variables, such as ethnicity or socioeconomic status, were generally well documented. However, with the exception of two trials (Silverman et al., 1999a,b), most studies had extremely low rates of ethnic minority participation (see Pina et al. [2003] who examined the differential treatment response of Hispanic/Latino youth and EuropeanAmerican youth). A noted strength of the cited investigations was the clinical severity of the research sample. All studies focused on subjects who sought clinical services and whose impairment was severe enough to warrant a psychiatric diagnosis. No study included children who were simply endorsing symptoms of anxiety on a self-report measure.

Outcome Measures. To their credit, the majority of cited investigations relied on a multimethod (e.g., clinical interview, self-report measures), multiinformant (e.g., child, parent, clinician) approach to document treatment outcomes. Both scalar and dichotomous measures that sampled specific symptom domains were regularly reported. Another strength of many of the cited investigations was that outcomes were not restricted to the simple reporting of statistically significant symptom improvement or symptom change. More clinically informative outcomes were commonly reported, such as clinically significant improvement (defined as changes that return deviant subjects to within nondeviant limits [Kendall and Grove, 1988]) and posttreatment diagnostic status (defined as the percentage of children who no longer meet criteria for a current anxiety disorder). For instance, 14 of the 21 investigations reported the posttreatment diagnostic status of subjects. However, the methods used to quantify diagnostic status varied moderately from study to study, which made it difficult to compare outcomes across trials. For instance, some studies combined information obtained from separate child and parent clinical interviews to determine posttreatment diagnostic status (Barrett, 1998; Barrett et al., 1996; Beidel et al., 2000; Last et al., 1998; Nauta et al., 2003; Silverman et al., 1999a,b), whereas others relied solely on 
information obtained from the parent (Cobham et al., 1998; Flannery-Schroeder and Kendall, 2000; Kendall, 1994; Kendall et al., 1997; Shortt et al., 2001; Spence et al., 2000). Moreover, some studies defined a subject as diagnosis free if criteria for his or her primary anxiety diagnosis were no longer met (Last et al., 1998), whereas others used a more restrictive definition and defined a subject as diagnosis free if criteria for both his or her primary and secondary (if present) anxiety diagnoses were no longer met (e.g., Barrett, 1998).

Moderators of Outcome. Ten of the 21 cited investigations reported results of secondary analyses that attempted to determine whether basic demographic and clinical variables moderated treatment outcome (e.g., age, sex, ethnicity, clinical severity, pretreatment diagnosis, comorbidities). The most frequent finding is that none of the variables analyzed moderate treatment outcome (for a notable exception, see Barrett et al. [1996]). However, the strength of this conclusion must be tempered because few studies were sufficiently powered to adequately address this important question.

Long-Term Follow-up. Although the follow-up period varied widely across the cited investigations (from 3 months to 6 years, with a modal length of 12 months), the general conclusion that can be reached is that CBT for anxiety disorders in children and adolescents is a durable intervention (Table 2). With few exceptions (Cobham et al., 1998), posttreatment gains were largely maintained at follow-up and showed little deterioration. Interestingly, several studies that found significant differences between two active treatments post-treatment reported that, at follow-up, the two treatments were equally effective. However, because all cited studies lacked an adequate control group during the follow-up period, competing explanations for the positive results reported cannot be dismissed.

\section{Treatments}

The behavioral treatment of fear and anxiety in children builds on early studies indicating that anxiety is readily conceptualized as a set of classically conditioned responses that can be unlearned or counterconditioned through associative pairing with anxiety-incompatible stimuli and responses. For example, in systematic desensitization (SD), anxiety-arousing stimuli are systematically and gradually paired (imaginally or in vivo) with competing stimuli such as food, praise, imagery, or cues generated from muscular relaxation. SD with children consists of three basic steps: (1) training in progressive muscle relaxation, (2) rank ordering of fearful situations from lowest to highest, and (3) hierarchical presentation of fear stimuli via imagery while the child is in a relaxed state (Eisen and Kearney, 1995). SD appears to work well with older children and adolescents. Younger children, however, often have difficulty with both obtaining vivid imagery and acquiring the incompatible muscular relaxation. Strategies such as using developmentally appropriate imagery and adjunctive use of workbooks may boost the effectiveness of these procedures with younger children.

Without encouragement, anxious children and adolescents often find it difficult to remain in the presence of anxiety-arousing stimuli for a sufficient length of time to allow habituation to occur in the natural environment. In fact, in some cases, the process of negative reinforcement maintains the anxiety response. That is, when an individual initially confronts an anxiety-provoking situation (e.g., the assignment of an oral report for the socially anxious youth), there is an increase in discomforting sensations and anxious thoughts (e.g., rapid heart rate, sweating, thoughts such as "I'll look stupid to others"). By escaping or avoiding the situation, such as through complaints of feeling ill and needing to leave class or the behavior of school avoidance/refusal, the individual feels immediate relief from the anxiety. This is the process of negative reinforcement. The escape behavior is reinforced by the relief and sets the stage for cycles of anxiety arousal followed by escape or avoidance and relief.

After the adult treatment literature, the identification of the negative reinforcement paradigm led to the development of exposure-based interventions for a wide range of pediatric anxiety disorders. Because escape and avoidance behaviors are negatively reinforced by the cessation of anxiety, exposure-based procedures require extended presentation of fear stimuli with concurrent prevention of escape and avoidance behaviors in order for the extinction of the conditioned responses to occur. Unlike systematic desensitization, stimulus presentation is not accompanied by progressive muscle relaxation. Rather, graduated imaginal and/or in vivo exposure to hierarchically presented fear stimuli is used to attenuate anxiety to phobic stimuli. Gradual exposure, with the consent of the child, is generally considered to produce less stress for the client (and therapist) and thus is often preferred over the use of more pre- 
COMPTON ET AL.

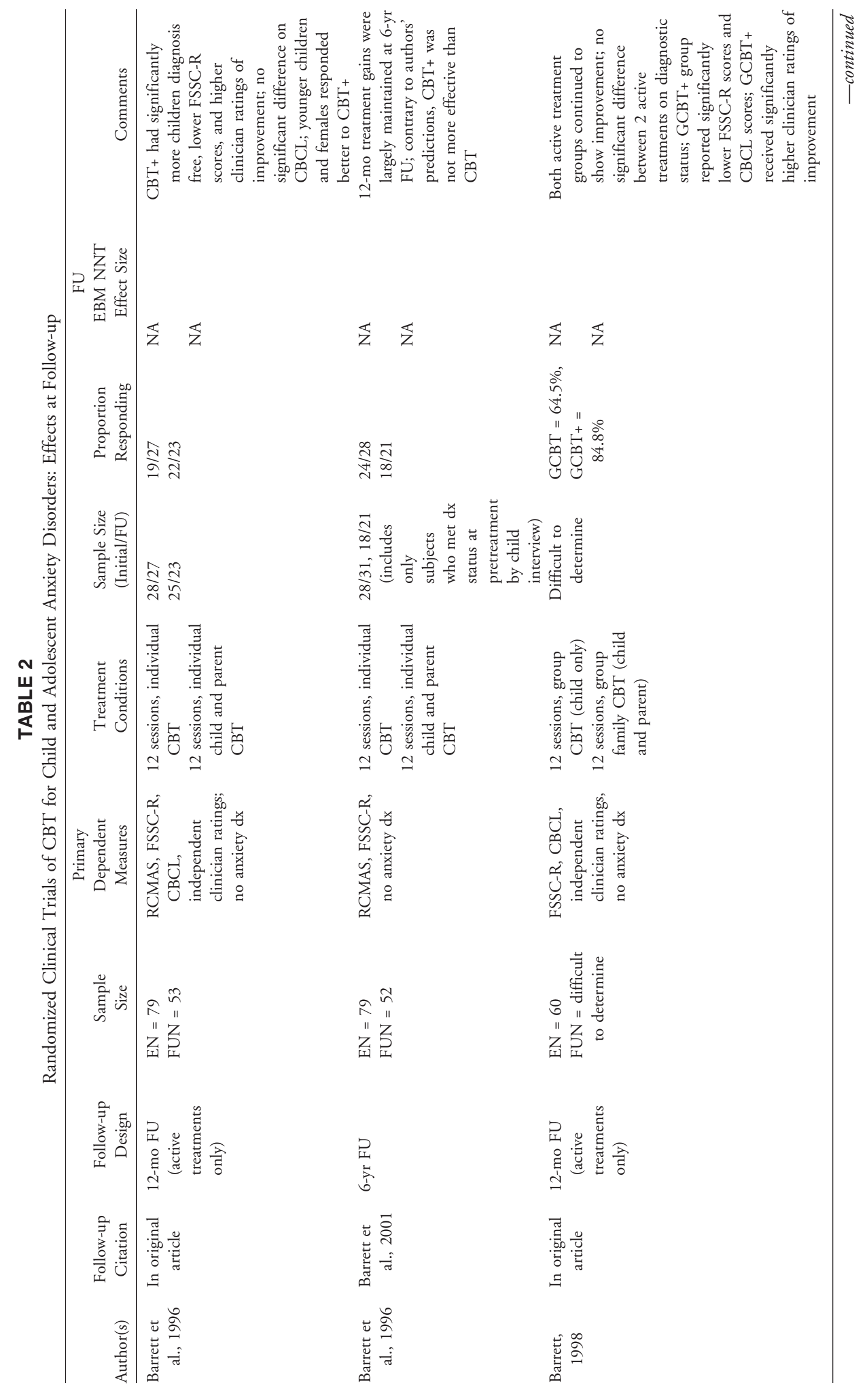


RESEARCH UPDATE REVIEW: CBT

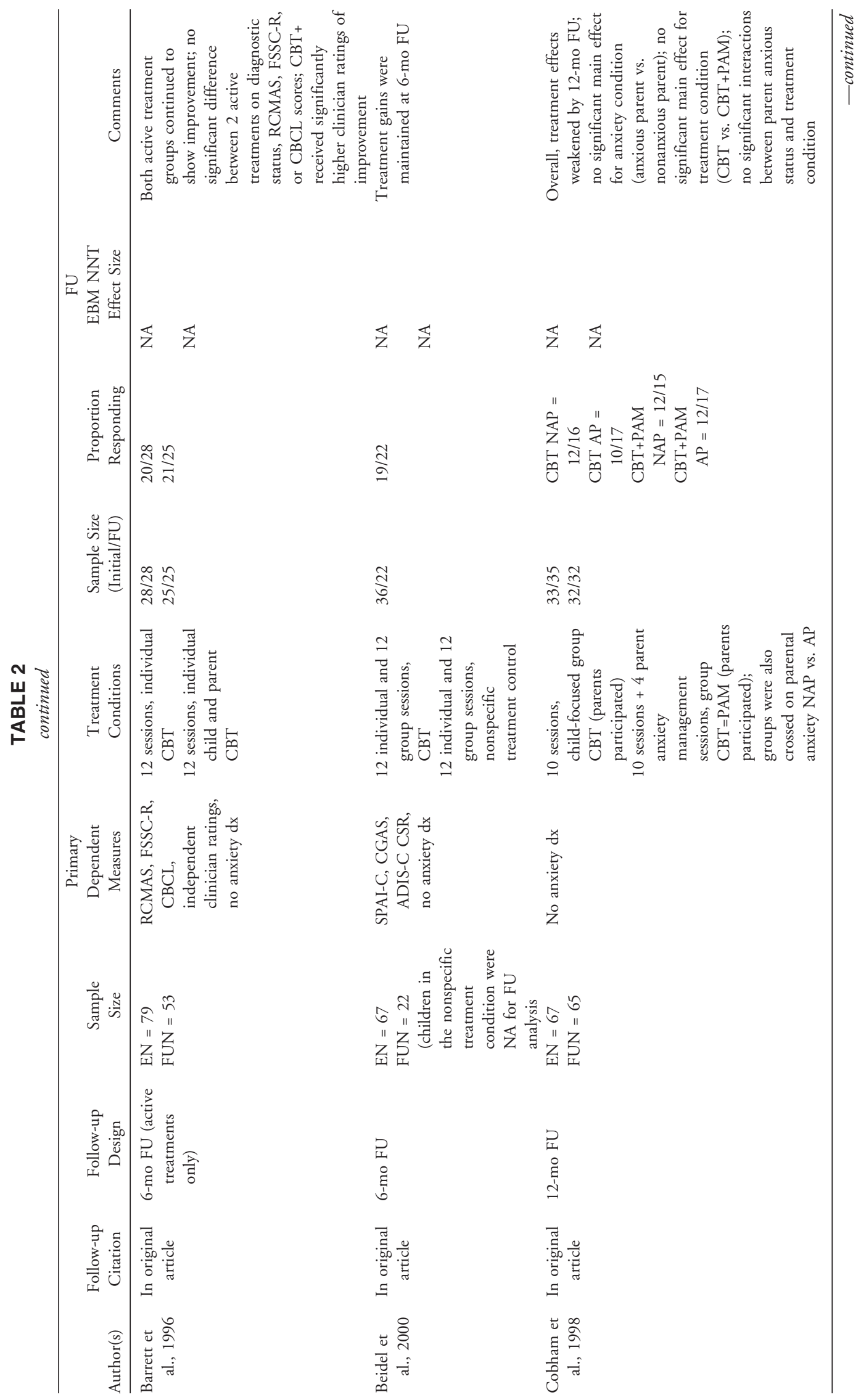




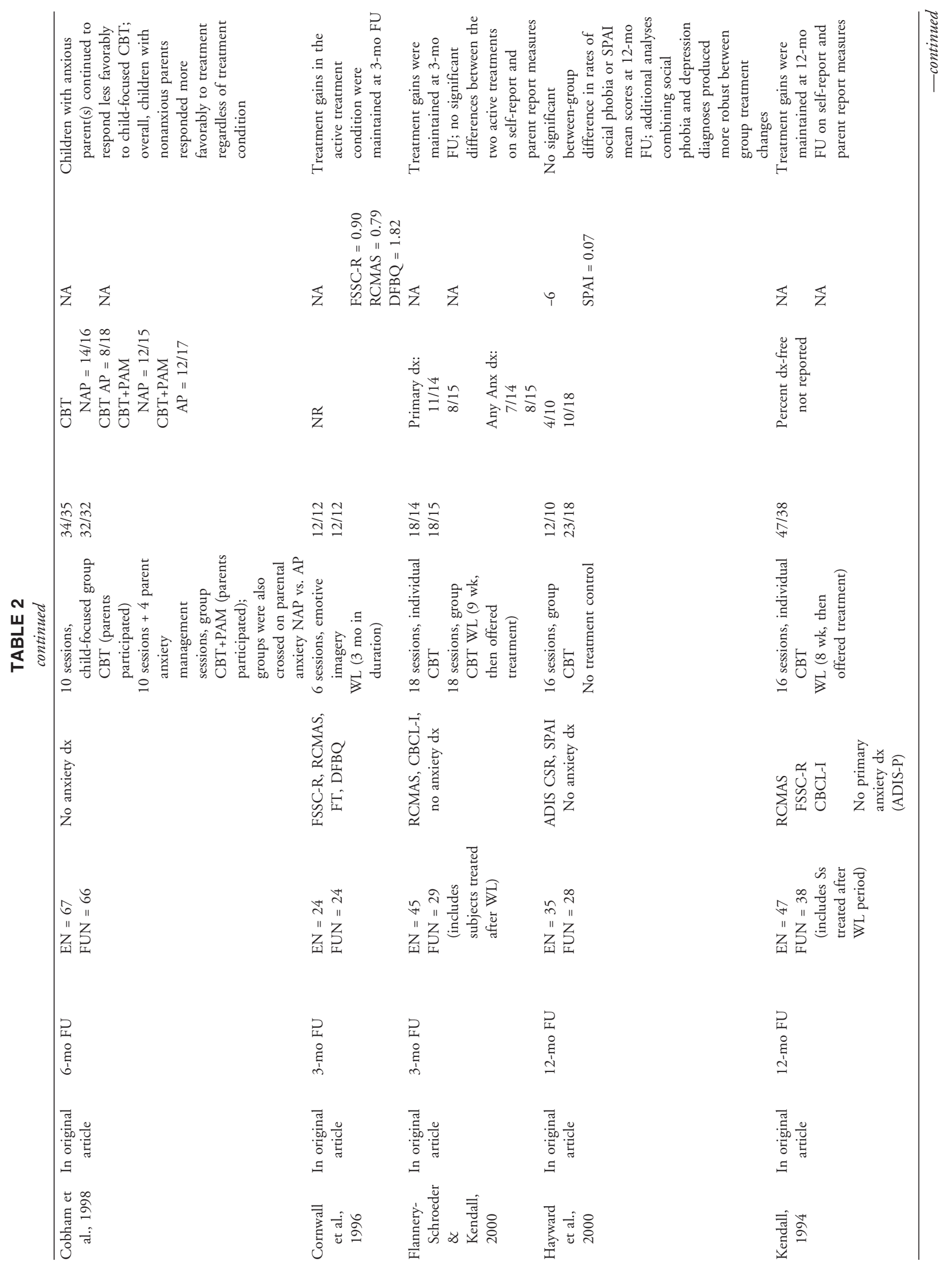


RESEARCH UPDATE REVIEW: CBT

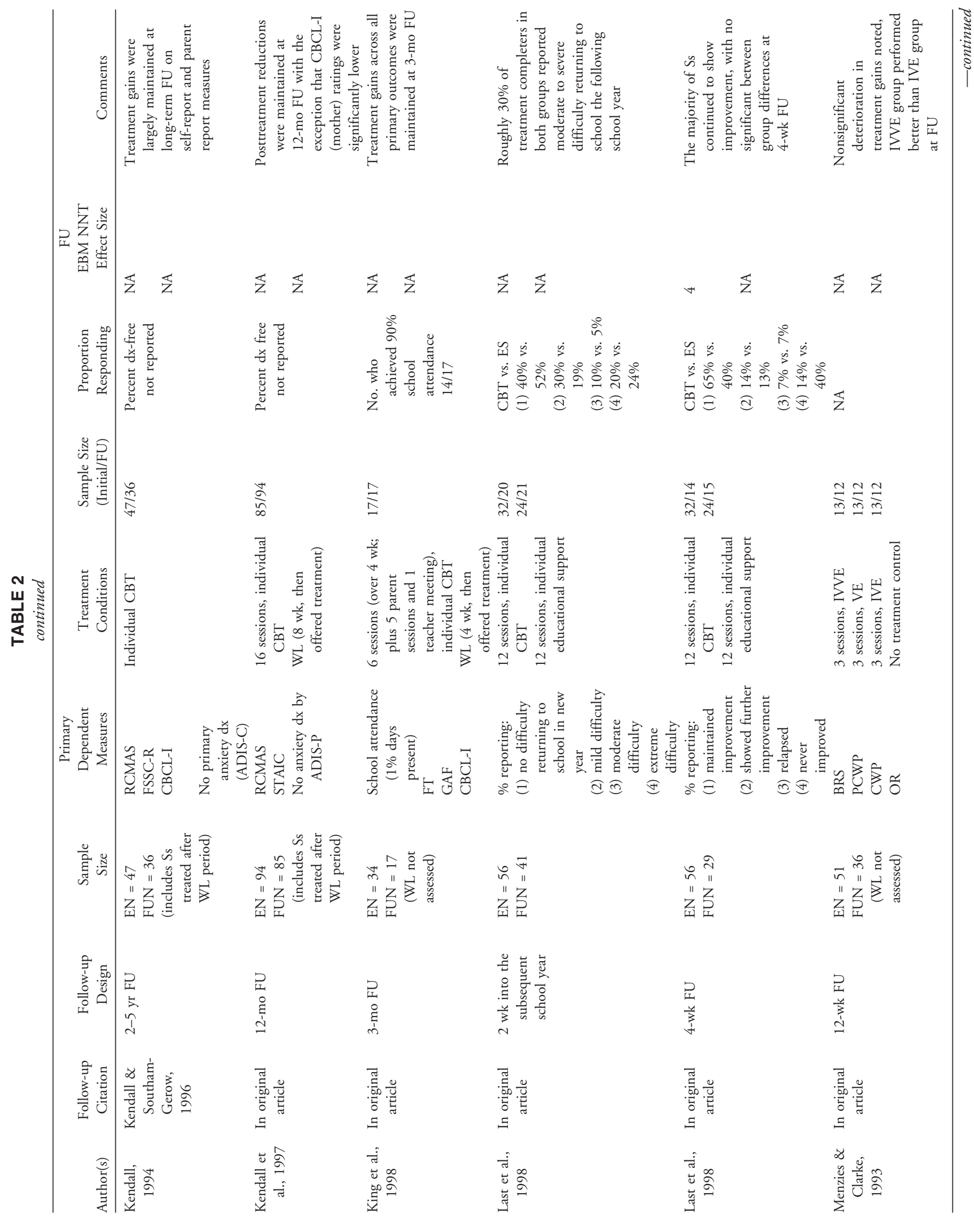




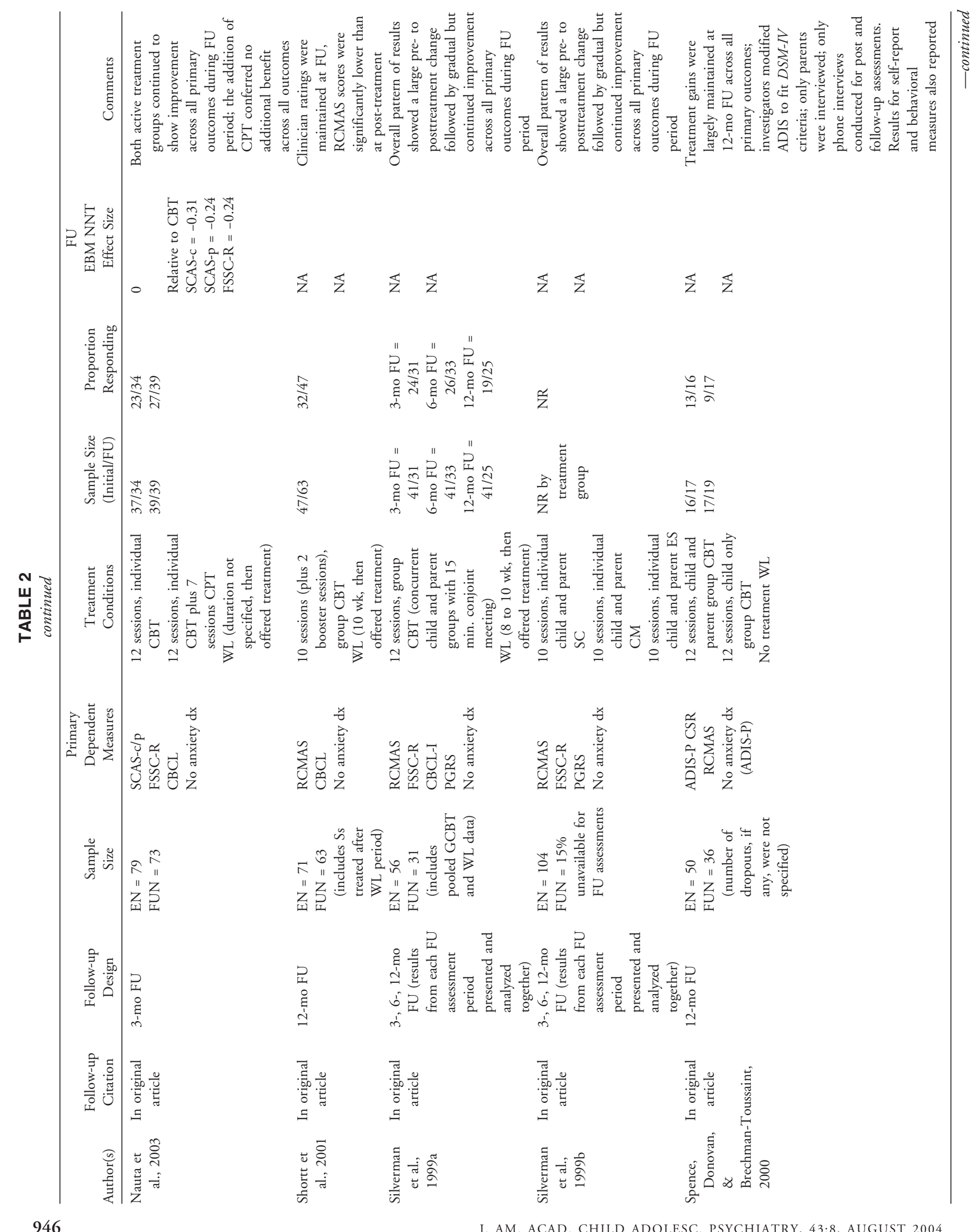




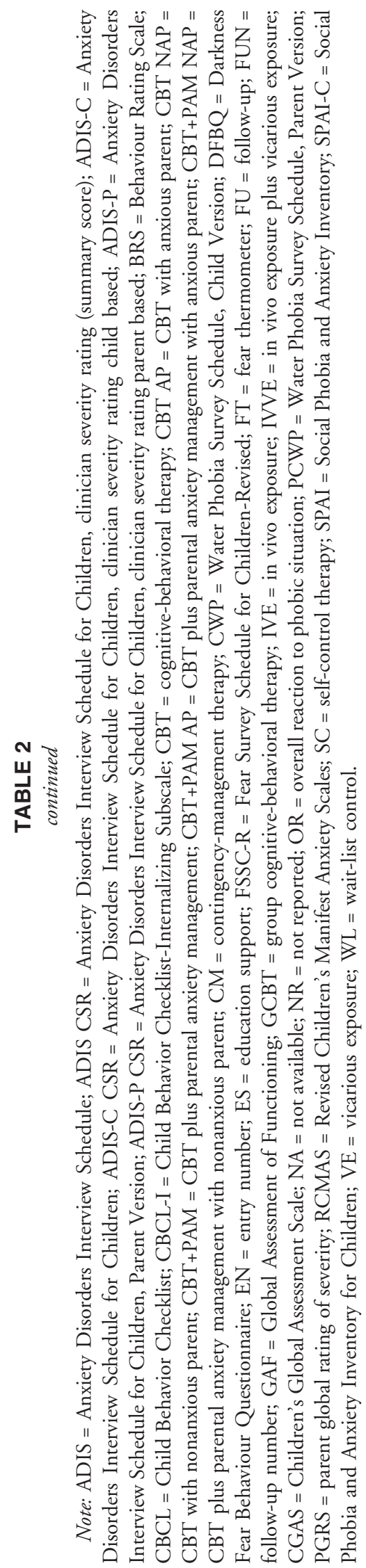

scriptive techniques, especially massed exposure or flooding.

Cognitive interventions, usually combined with exposure, also play a prominent role in CBT for anxious children and adolescents. For example, Kendall and colleagues developed a comprehensive cognitivebehavioral protocol for anxious youth that focuses on transmitting coping skills to children in need (Kendall, 1994; Kendall et al., 1997). Based on the premise that anxious children view the world through a "template" of threat, automatic questioning (e.g., "What if ..."), and behavioral avoidance, treatment is focused on providing educational experiences to build a new "coping template" for the child. Therapists assist the children to reconceptualize anxiety-provoking situations as problems to be solved and situations with which to cope. A variety of cognitive-behavioral components assist the therapist and child in building the coping template: relaxation training, imagery, correcting maladaptive self-talk, problem-solving skills, and managing reinforcers. Therapists use coping modeling, role-play rehearsals, in vivo exposure, and a collaborative therapeutic relationship with the child to facilitate the treatment progress. As a rule, parents are actively involved in all facets of treatment as collaborators in the change process.

For example, when significant others are trapped in the child's anxiety symptoms, it is crucial that they stop participating in or reinforcing the child's avoidance strategies or rituals. To test the hypothesis that adding a family anxiety management component would boost treatment effectiveness, Barrett et al. (1996) developed a parallel family program to Kendall's "Coping Cat" based on behavioral family intervention strategies found effective for the treatment of externalizing disorders in youth. After the completion of each child session with the therapist, the child and parents would participate in a family anxiety management session with the therapist. The crux of the program is to empower parents and children by forming an "expert team" to overcome and master anxiety. Parents are trained in reinforcement strategies, with an emphasis on differential reinforcement and systematic ignoring of excessive complaining and anxious behavior. However, unilateral extinction strategies, such as when a parent returns the school-phobic child to school by force, have significant disadvantages relative to consensual child involvement: (1) lack of a workable strategy 
for managing the child's distress, (2) disruption of the treatment relationship, (3) inability to target symptoms that are out-of-sight for parents and teachers, and (4), most important, failure to help the child internalize a more skillful strategy for coping with current and potential future anxiety symptomatology.

\section{MAJOR DEPRESSION}

At any one time, approximately 1 in 20 children and adolescents suffers from major depressive disorder, with rates of depression rising dramatically in adolescents, especially in girls. Although the economic burden of depression in youth is uncertain, the human burden is considerable, especially with teenage suicide. Hence, it is of critical importance to note that the empirical literature is more supportive for problem-specific psychotherapies, especially CBT, than for medication management of pediatric depressive disorders (Birmaher et al., 1996a; Hoberman et al., 1996). In particular, several controlled trials have demonstrated that individual or group administered cognitive-behavioral psychotherapy is an effective treatment for depressed youth (Brent et al., 1997; Lewinsohn et al., 1994), and some investigators now consider CBT to be the treatment of choice for this disorder (Reinecke et al., 1998).

\section{Types of Investigations}

Twelve articles describing a variety of cognitivebehavioral intervention packages for the treatment of child and adolescent depression were identified (Table 3). Although these depression trials are equally methodologically rigorous when compared with child and adolescent anxiety trials (e.g., contrasting group designs comparing one or more active treatments with either no treatment, wait-list, or attention placebo controls), the number of studies is significantly fewer, and the research agenda to date has been less coherent and systematic. Moreover, several of the studies with null findings likely had insufficient power to detect a between-group treatment effect due to the small sample size of each treatment condition. This is a notable deficiency and contributes to the widely held notion among practitioners that all treatments for depression are equally effective. It also makes it difficult, if not impossible, to reach strong conclusions regarding the differential efficacy of the treatments evaluated.

Two studies addressed the question of whether adding a separate treatment module for parents incremen- tally improves outcomes (Clarke et al., 1999; Lewinsohn et al., 1990). One study compared individual CBT to systemic behavioral family therapy (Brent et al., 1997). Another study evaluated the relevant question of whether adding CBT to usual care in a health maintenance organization is better than usual care alone (Clarke et al., 2002). Five studies evaluated the efficacy of one or more CBT interventions in designs that included either an attention placebo condition (Kahn et al., 1990; Liddle and Spence, 1990; Vostanis et al., 1996; Wood et al., 1996) or a notreatment control (Weisz et al., 1997). One study compared individual CBT with interpersonal psychotherapy (Rossello and Bernal, 1999). One investigation evaluated the effects of maintenance CBT for depressed adolescents (Clarke et al., 1999). One study evaluated the acceptability and efficacy of a combined cognitivebehavioral family education treatment (Asarnow et al., 2002). Finally, one study evaluated the efficacy of cognitive bibliotherapy for adolescents with mild to moderate depressive symptoms (Ackerson et al., 1998). No published investigations compared components of treatments, and there were no systematic replication studies by independent investigators.

\section{Assessment Issues}

Diagnosis and Symptom Profile. Six of the 12 studies used semistructured clinical interviews to identify subjects as having DSM major depressive disorder or dysthymia (Brent et al., 1997; Clarke et al., 1999, 2002; Lewinsohn et al., 1990; Vostanis et al., 1996; Wood et al., 1996). The most commonly used interview was the Schedule for Affective Disorders and Schizophrenia for School-Age Children (Chambers et al., 1985; Orvaschel and Puig-Antich, 1986; Puig-Antich and Chambers, 1978). The remaining six studies either failed to mention the specific assessment procedures used to determine inclusion criteria (Rossello and Bernal, 1999) or enrolled subjects solely on the basis of mild to moderate levels of self-reported depressive symptomatology (Ackerson et al., 1998; Asarnow et al., 2002; Kahn et al., 1990; Liddle and Spence, 1990; Weisz et al., 1997). The same six investigations that used semistructured clinical interviews also assessed comorbidity but failed to analyze whether comorbidity status was related to treatment outcome. Thus, failure to systematically assess the impact of comorbidity on outcome is a critical deficiency in both the anxiety and depression literature. 


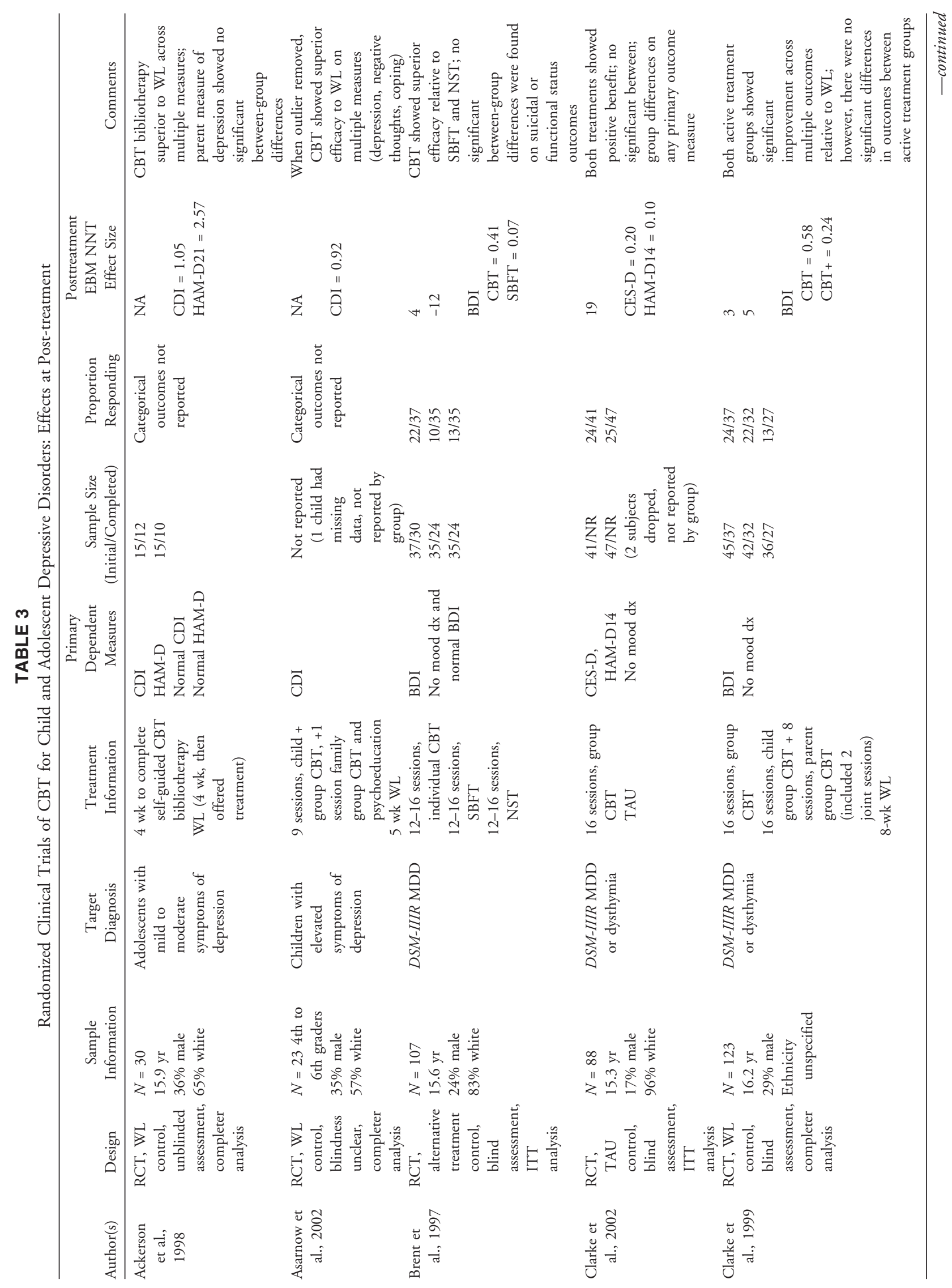




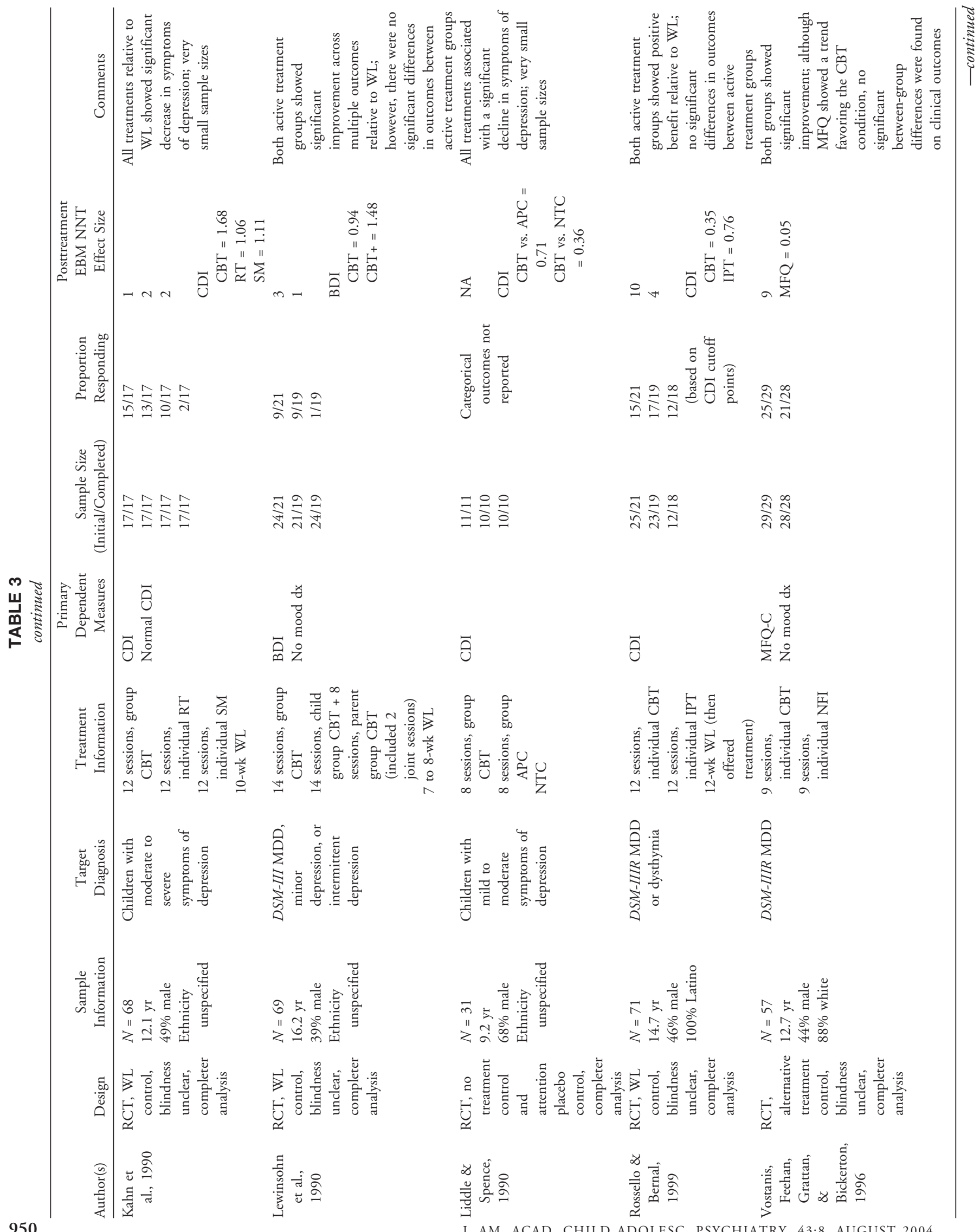




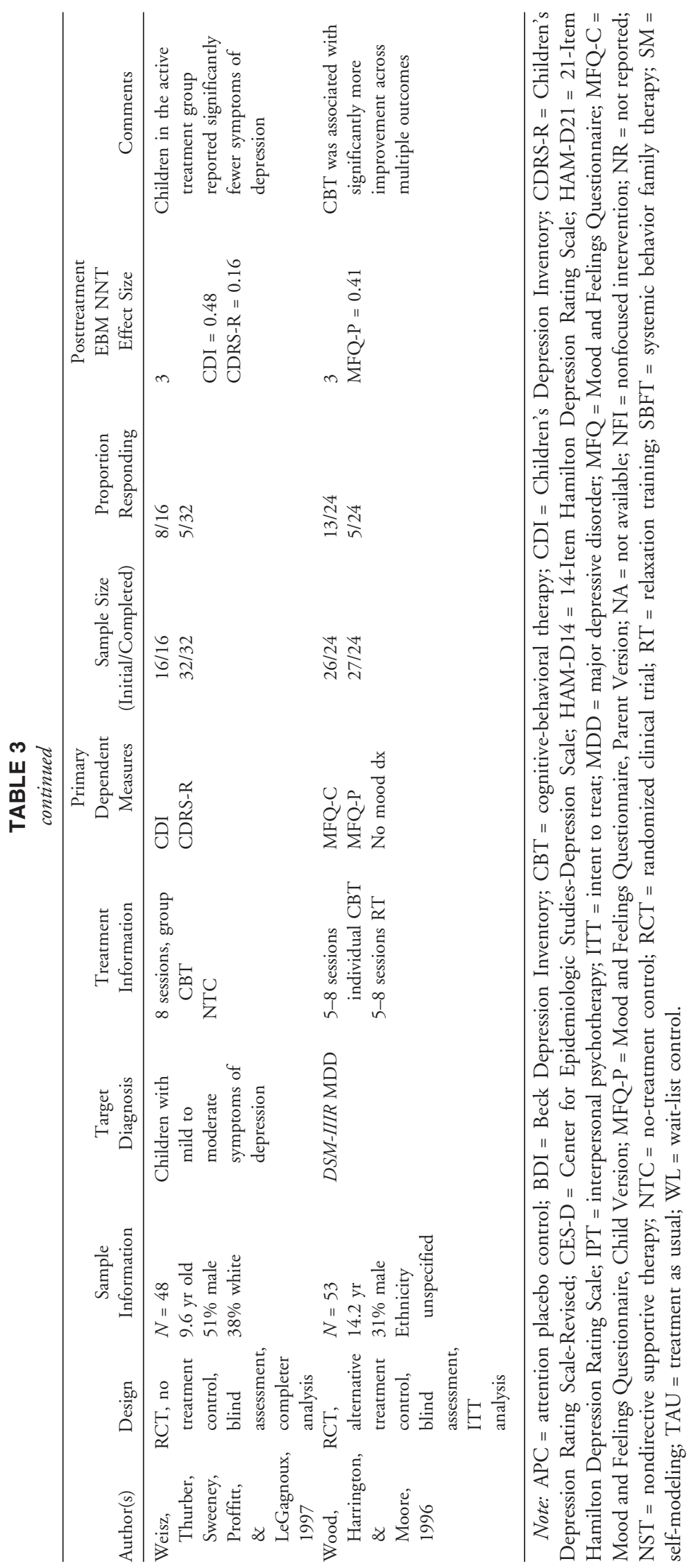


Demographics. Although both males and females are represented in the treated populations, other basic demographic variables, such as ethnicity, were generally not well documented. Only 7 of the 12 cited investigations provided the ethnic breakdown of the sample. Moreover, the majority of children who have participated in research studies to date have been overwhelmingly white, suggesting that future studies will be needed to evaluate the exportability of protocol-driven CBT treatment packages to divergent patient populations.

Outcome Measures. All the cited investigations relied on psychometrically sound measures to document treatment results and changes in specific symptom domains. One-half of the studies reported the percentage of subjects who no longer met criteria for a depressive disorder after treatment (Brent et al., 1997; Clarke et al., 1999, 2002; Lewinsohn et al., 1990; Vostanis et al., 1996; Wood et al., 1996), and several studies reported the percentage of subjects who returned to the nondeviant ranges on the primary outcome measures. Only three investigations provided quantitative measures of change in functional status (Brent et al., 1997; Clarke et al., 1999, 2002). Because little is currently known about how treatments affect academic, social, and family domains, future studies would benefit from including a more diverse range of outcomes (Compton et al., 2002).

Long-Term Follow-up. The data addressing the durability of CBT for adolescent depression are mixed (Table 4). In general, studies characterized by a relatively short follow-up period (from 1 to 9 months) report that posttreatment gains are largely maintained, with several studies showing continued improvement. However, studies with longer follow-up periods (from 9 months to 2 years) and low attrition rates at followup found that a sizable percentage of subjects continued to report significant depressive symptoms or a recurrence of their depressive illness (Birmaher et al., 2000; Vostanis et al., 1996, 1998; Wood et al., 1996). Factors found to predict a lack of recovery or relapse include low self-esteem (Vostanis et al., 1996, 1998), comorbidity at post-treatment (Vostanis et al., 1998), severity of depression or high level of functional impairment at baseline (Birmaher et al., 2000), the presence of subsyndromal depression (Brent et al., 2001), parental depression (Brent et al., 1998; Clarke et al., 2002), parent-child conflict (Birmaher et al., 2000), and the source of treatment referral (Birmaher et al., 2000). These studies suggest that depression in adolescence is associated with a high risk of recurrence. They also underscore the importance of developing interventions that specifically target adolescents at risk of relapse and investigate the impact of continuation treatment on long-term outcomes.

\section{Treatments}

Like other cognitive-behavioral treatment packages, CBT for depression in youths is a present-oriented, skills-based treatment that, in this case, is based on the assumption that depression is either caused or maintained by the way one perceives situations and events (e.g., cognitions about the world and self) and the presence of skill deficits (both emotional and behavioral) that prevent the patient from interacting effectively with the world. Because personality is an interactive multidirectional system of cognitions, behaviors, and emotions, depression is manifested in each of the three components of the personality. However, CBT for depression assumes that symptom change is most likely to occur through interventions that modify patterns of behavior through skills acquisition and patterns of cognition, with changes in depressed mood following in turn. Among the behavioral and cognitive skill deficits that may characterize a depressed youth are low levels of involvement in pleasant activities, poor problemsolving and assertion skills, cognitive distortions that negatively bias perceptions, negative automatic thoughts, negative views of self and future, and failure to attribute positive outcomes to internal, stable, or global causes. The role of the therapist, therefore, is to establish a collaborative working relationship with the adolescent and to help the adolescent learn new ways of behaving and thinking, which in turn reduces depressive severity and risk of relapse.

Current cognitive-behavioral treatment packages for depressed youths share two salient characteristics: (1) general and "required" skill building sessions and optional "modular" sessions for specific problems and (2) the integration of parent and family sessions with individual CBT (Treatment for Adolescents with Depression Study, 2003). Treatment is generally designed to improve the teenager's problem-solving ability when faced with a stressful situation, for example, parent-child conflict, role transitions, grief reactions, or peer problems. Therefore, the required aspects of 


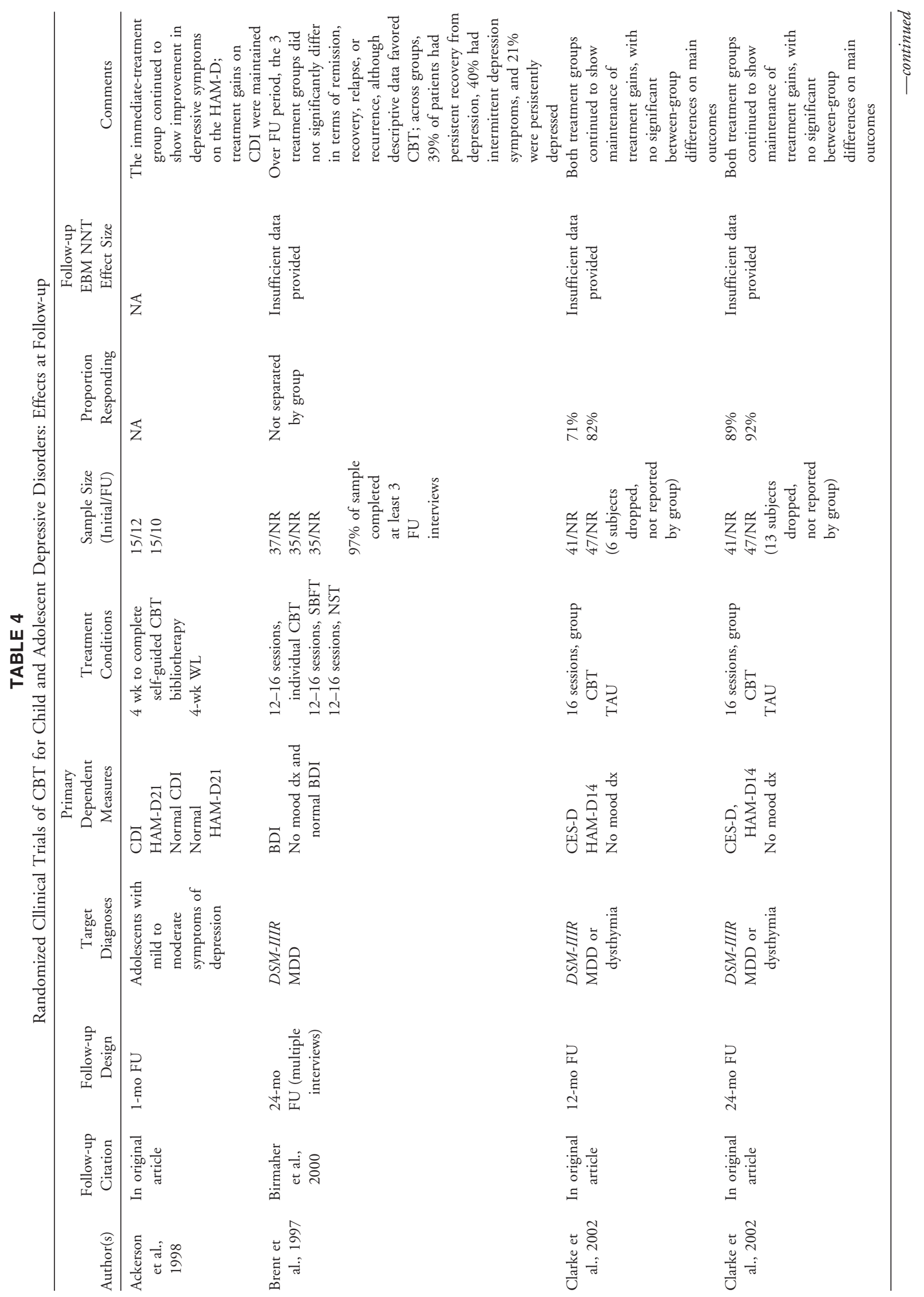


COMPTON ET AL.

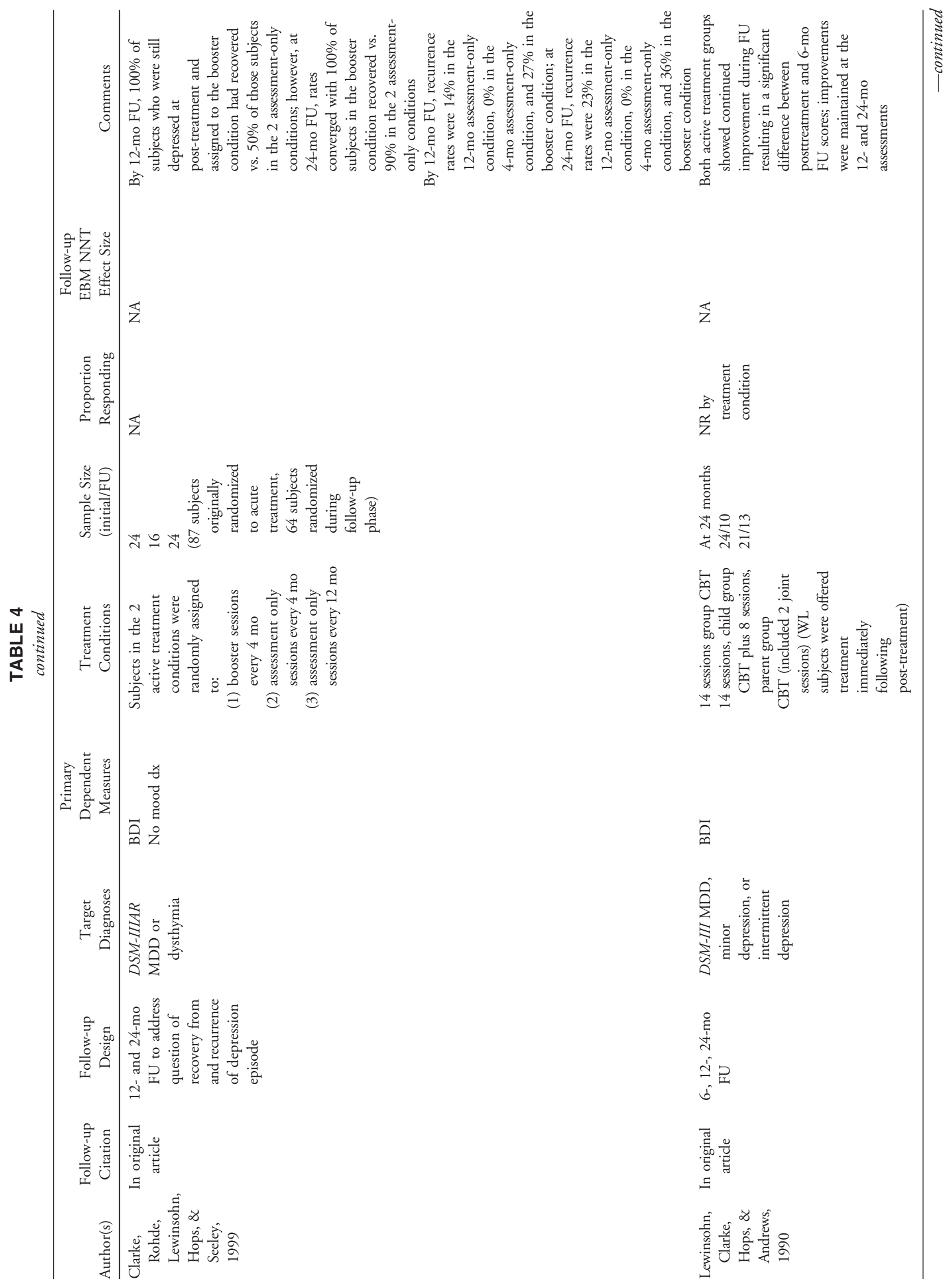




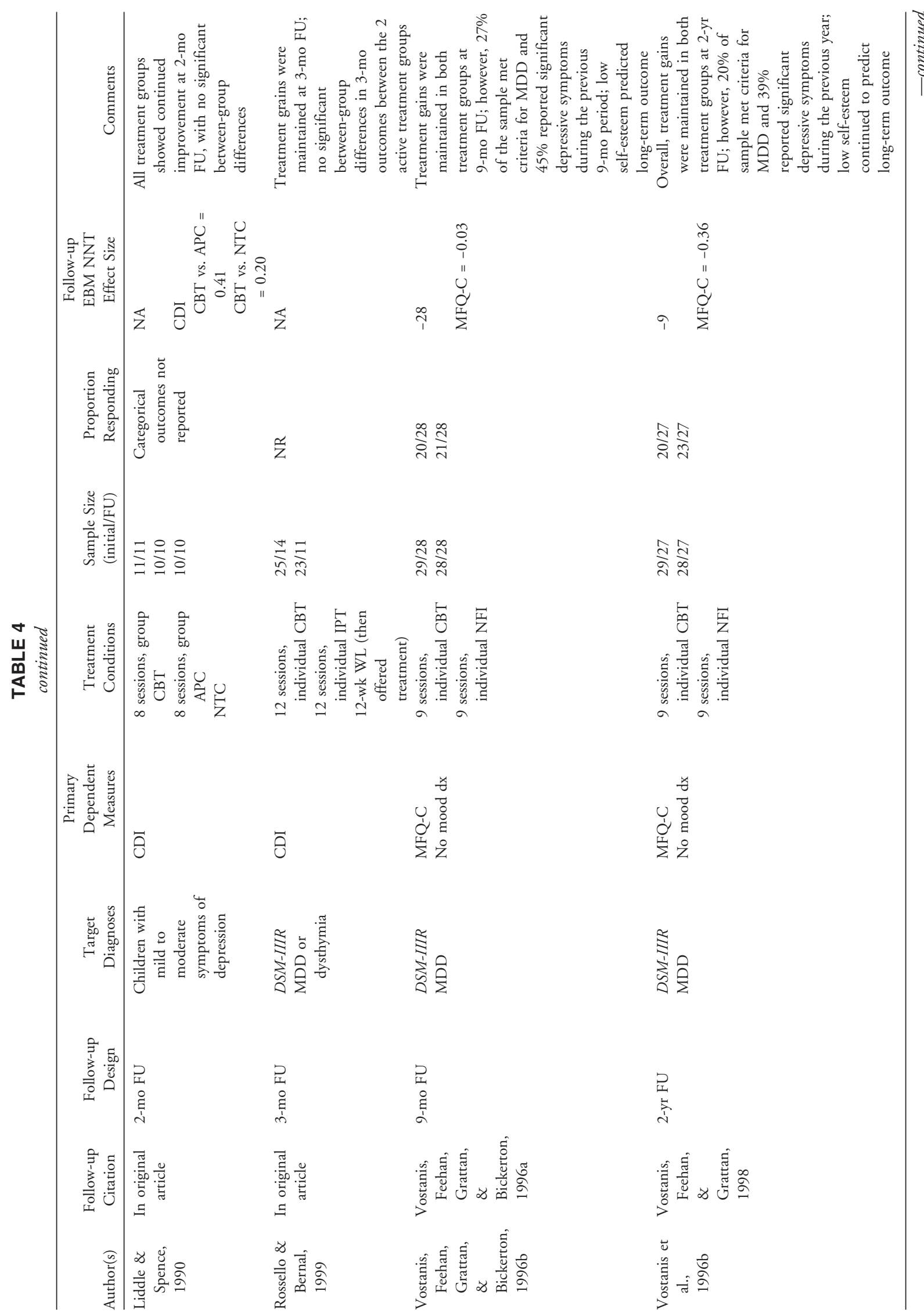




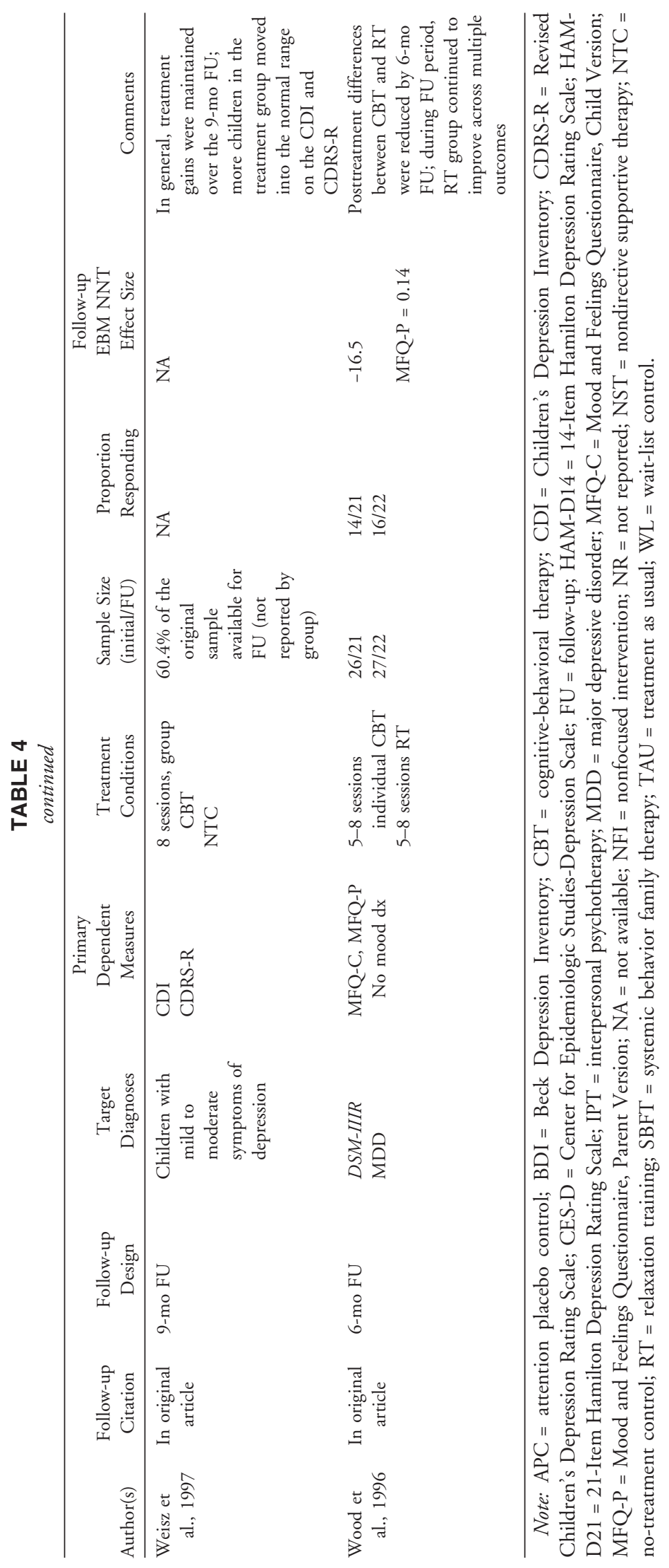


treatment include psychoeducation about depression and its causes, goal setting with the adolescent, and general problem-solving skills. Modules, chosen jointly by the therapist and adolescent, then address the specific skill deficits of the teenager. Because parentchild conflict is both a risk factor for depression and predictive of poor treatment outcome and relapse, including a parent component in CBT is justified on an ad hoc basis. Moreover, evidence is beginning to emerge that combined child and parent treatment may be more effective than treatment directed at the teenager alone (Lewinsohn et al., 1990). In addition to teaching contingency management procedures, parents are provided with alternative, effective methods for parenting and creating a more positive family environment. Furthermore, family interactions are targeted directly to shape and reinforce effective communication and to increase pleasant activities and positive affect.

\section{DISCUSSION}

A substantial evidence base supports the efficacy of problem-specific cognitive-behavioral interventions for a variety of childhood and adolescent anxiety and depressive disorders. Unlike other psychotherapeutic techniques that have been applied to these disorders, CBT is consistent with an EBM perspective that values empirically supported problem-focused treatments. CBT presents a logical theoretical framework to guide practitioners through an idiographic assessment of specific problem domains, the delivery of problem-specific treatment interventions, and well-specified outcomes to monitor treatment progress. However, CBT is not simplistic. Helping children, adolescents, and parents make rapid and difficult behavior change over short time intervals requires considerable expertise and training.

Future research in the areas of childhood and adolescent anxiety and depressive disorders will need to focus on the following areas. First, controlled trials comparing medications, CBT, and their combination are needed to determine whether combined treatment provides an additive benefit in terms symptom reduction. Second, treatment-dismantling studies are needed to identify the relative contributions of specific CBT components to symptom reduction and treatment acceptability. Third, mediational analyses (how a treat- ment works) are needed to refine treatment interventions and better understand the mechanism(s) through which treatments achieve their therapeutic effect. Fourth, follow-up studies with adequate control groups will be necessary to evaluate the long-term benefit of CBT, including examining whether booster CBT sessions reduce relapse rates and whether intervening in childhood prevents the onset of adult psychiatric disorders. Finally, studies with diverse patient populations are needed to evaluate the exportability and generalizability of currently available protocoldriven treatments.

Disclosure: Dr. March receives consulting fees from Solvay, Pfizer, GSKI, Wyeth-Ayerst, and BMS and serves as Scientific Advisor for Shire and Pfizer and DSMB Chair for the Organon Study. He has financial relationships with MultiHealth Systems for the Multidimensional Anxiety Scale for Children.

\section{REFERENCES}

Ackerson J, Scogin F, McKendree-Smith N, Lyman RD (1998), Cognitive bibliotherapy for mild and moderate adolescent depressive symptomatology. J Consult Clin Psychol 66:685-690

Albano AM, Silverman WK (1996), Clinician's Guide to the Anxiety Disorders Interview Schedule for DSM-IV, Child Version. Boulder, CO: Graywind Publications

Andreason N (1997), Linking mind and brain in the study of mental illnesses: a project for a scientific psychopathology. Science 275:15861598

Asarnow JR, Scott CV, Mintz J (2002), A combined cognitive-behavioral family education intervention for depression in children: a treatment development study. Cognit Ther Res 26:221-229

Barlow DH (1993), On the relation of clinical research to clinical practice: current issues, new directions. In: Issues in Clinical Psychology: The Relationship Between the Scientist and the Practitioner, Mindell J, ed. Dubuque, IA: Wm. C. Brown, pp 11-35

Barrett PM (1998), Evaluation of cognitive-behavioral group treatments for childhood anxiety disorders. J Clin Child Psychol 27:459-468

Barrett PM, Dadds MR, Rapee RM (1996), Family treatment of childhood anxiety: a controlled trial. J Consult Clin Psychol 64:333-342

Barrett PM, Duffy AL, Dadds MR, Rapee RM (2001), Cognitivebehavioral treatment of anxiety disorders in children: long-term (6-year) follow-up. J Consult Clin Psychol 69:135-141

Beidel DC, Turner SM, Morris TL (2000), Behavioral treatment of childhood social phobia. J Consult Clin Psychol 68:1072-1080

Bernstein GA, Shaw K (1997), Practice parameters for the assessment and treatment of children and adolescents with anxiety disorders. J Am Acad Child Adolesc Psychiatry 36:69S-84S

Birmaher B, Brent DA, Kolko D et al. (2000), Clinical outcome after short-term psychotherapy for adolescents with major depressive disorder. Arch Gen Psychiatry 57:29-36

Birmaher B, Ryan ND, Williamson DE, Brent DA, Kaufman J (1996a), Childhood and adolescent depression: a review of the past 10 years. Part II. J Am Acad Child Adolesc Psychiatry 35:1575-1583

Birmaher B, Ryan ND, Williamson DE et al. (1996b), Childhood and adolescent depression: a review of the past 10 years. Part I. J Am Acad Child Adolesc Psychiatry 35:1427-1439

Brent DA, Birmaher B, Kolko D, Baugher M, Bridge J (2001), Subsyn- 
dromal depression in adolescents after a brief psychotherapy trial: course and outcome. J Affect Disord 63:51-58

Brent DA, Holder D, Kolko D et al. (1997), A clinical psychotherapy trial for adolescent depression comparing cognitive, family, and supportive therapy. Arch Gen Psychiatry 54:877-885

Brent DA, Kolko DJ, Birmaher B et al. (1998), Predictors of treatment efficacy in a clinical trial of three psychosocial treatments for adolescent depression. J Am Acad Child Adolesc Psychiatry 37:906-914

Chambers WJ, Puig-Antich J, Hirsch M et al. (1985), The assessment of affective disorders in children and adolescents by semistructured interview. Test-retest reliability of the schedule for affective disorders and schizophrenia for school-age children, present episode version. Arch Gen Psychiatry 42:696-702

Clarke G, Lewinsohn P, Hops H (1990), Instructor's Manual for the Adolescent Coping With Depression Course, 4th ed. Eugene, OR: Castalia Press

Clarke GN, Hornbrook M, Lynch F et al. (2002), Group cognitivebehavioral treatment for depressed adolescent offspring of depressed parents in a health maintenance organization. J Am Acad Child Adolesc Psychiatry 41:305-313

Clarke GN, Rohde P, Lewinsohn PM, Hops H, Seeley JR (1999), Cognitive-behavioral treatment of adolescent depression: efficacy of acute group treatment and booster sessions. J Am Acad Child Adolesc Psychiatry 38:272-279

Cobham VE, Dadds MR, Spence SH (1998), The role of parental anxiety in the treatment of childhood anxiety. J Consult Clin Psychol 66:893905

Cohen J, March J, Berliner L (2000), Treatment of PTSD in Children and Adolescents. In: Effective Treatments for PTSD, Foa E, Davidson J, Keane T, eds. New York: Guilford, pp 106-138

Compton SN, Burns BJ, Egger HL, Robertson E (2002), Review of the evidence base for treatment of childhood psychopathology: internalizing disorders. J Consult Clin Psychol 70:1240-1266

Cornwall E, Spence SH, Schotte D (1996), The effectiveness of emotive imagery in the treatment of darkness phobia in children. Behav Change $13: 223-229$

Curry JF, Reinecke MA (2003), Modular therapy for adolescents with major depression. In: Cognitive Therapy With Children and Adolescents: A Casebook for Clinical Practice, 2nd ed., Reinecke MA, Dattilio FM et al., eds. New York: Guilford, pp 95-128

Eisen A, Kearney C (1995), Treating Fear and Anxiety in Children and Adolescents. Northvale, NJ: Jason Aronson

Flannery-Schroeder EC, Kendall PC (2000), Group and individual cognitive-behavioral treatments for youth with anxiety disorders: a randomized clinical trial. Cognit Ther Res 24:251-278

Franklin ME, Rynn M, March JS, Foa EB (2002), Obsessive-compulsive disorder. In: Clinical Behavior Therapy: Adults and Children, Hersen M, ed. New York: Wiley, pp 276-303

Geddes J, Reynolds S, Streiner D, Szatmari P (1997), Evidence based practice in mental health [editorial]. BMJ 315:1483-1484

Guyatt GH, Sackett DL, Cook DJ (1993), Users' guides to the medical literature. II. How to use an article about therapy or prevention. A. Are the results of the study valid? Evidence-Based Medicine Working Group. JAMA 270:2598-2601

Guyatt GH, Sackett DL, Cook DJ (1994), Users' guides to the medical literature. II. How to use an article about therapy or prevention. B. What were the results and will they help me in caring for my patients? Evidence-Based Medicine Working Group. JAMA 271:59-63

Guyatt GH, Sinclair J, Cook DJ, Glasziou P (1999), Users' guides to the medical literature: XVI. How to use a treatment recommendation. Evidence-Based Medicine Working Group and the Cochrane Applicability Methods Working Group. JAMA 281:1836-1843

Haynes SN, O'Brien WH (1990), Functional analysis in behavior therapy. Clin Psychol Rev 10:649-668

Hayward C, Varady S, Albano AM, Thienemann M, Henderson L, Schatzberg AF (2000), Cognitive-behavioral group therapy for social phobia in female adolescents: results of a pilot study. J Am Acad Child Adolesc Psychiatry 39:721-726

Hibbs E, Jensen P (1996), Psychosocial Treatments for Child and Adolescent Disorders Washington, DC: American Psychological Press
Hoberman HM, Clarke GN, Saunders SM (1996), Psychosocial interventions for adolescent depression: issues, evidence, and future directions. Prog Behav Modif 30:25-73

Hyman SE (2000), The millennium of mind, brain, and behavior. Arch Gen Psychiatry 57:88-89

Kahn JS, Kehle TJ, Jenson WR, Clark E (1990), Comparison of cognitivebehavioral, relaxation, and self-modeling interventions for depression among middle-school students. School Psychol Rev 19:196-211

Kendall P (1993), Guiding theory for therapy with children and adolescents. In: Child and Adolescent Therapy, Kendall P, ed. New York: Guilford, pp 3-22

Kendall PC (1994), Treating anxiety disorders in children: results of a randomized clinical trial. J Consult Clin Psychol 62:100-110

Kendall PC, Aschenbrand SG, Hudson JL (2003), Child-focused treatment of anxiety. In: Evidence-Based Psychotherapies for Children and Adolescents, Kazdin AE, ed. New York: Guilford, pp 81-100

Kendall PC, Chu BC, Pimentel SS, Choudhury M (2000), Treating anxiety disorders in youth. In: Child and Adolescent Therapy: CognitiveBehavioral Procedures, 2nd ed. Kendall PC, ed. New York: Guilford, pp 235-287

Kendall PC, Flannery-Schroeder E, Panichelli-Mindel SM, SouthamGerow M (1997), Therapy for youths with anxiety disorders: a second randomized clinical trial. J Consult Clin Psychol 65:366-380

Kendall PC, Grove WM (1988), Normative comparisons in therapy outcome. Behav Assess 10:147-158

Kendall PC, Krain A, Treadwell KR (1999), Generalized anxiety disorder. In: Handbook of Prescriptive Treatments for Children and Adolescents, 2nd ed. Ammerman RT, Hersen M, Last CG, eds. Boston: Allyn and Bacon, pp 155-171

Kendall PC, Panichelli-Mindel SM (1995), Cognitive-behavioral treatments. J Abnorm Child Psychol 23:107-124

Kendall PC, Southam-Gerow MA (1996), Long-term follow-up of a cognitive-behavioral therapy for anxiety-disordered youth. J Consult Clin Psychol 64:724-730

King NJ, Tonge BJ, Heyne D et al. (1998), Cognitive-behavioral treatment of school-refusing children: a controlled evaluation. J Am Acad Child Adolesc Psychiatry 37:395-403

Last CG, Hansen C, Franco N (1998), Cognitive-behavioral treatment of school phobia. J Am Acad Child Adolesc Psychiatry 37:404-411

Lewinsohn P, Clarke GN, Rohde P (1994), Psychological Approaches to the Treatment of Depression in Adolescents. New York: Plenum

Lewinsohn PM, Clarke GN, Hops H, Andrews JA (1990), Cognitivebehavioral treatment for depressed adolescents. Behav Ther 21:385401

Liddle B, Spence SH (1990), Cognitive-behaviour therapy with depressed primary school children: a cautionary note. Behav Psychother 18:85102

Manassis K, Mendlowitz SL, Scapillato D et al. (2002), Group and individual cognitive-behavioral therapy for childhood anxiety disorders. A randomized trial. J Am Acad Child Adolesc Psychiatry 41:1423-1430

March J, Mulle K (1998), OCD in Children and Adolescents: A CognitiveBehavioral Treatment Manual. New York: Guilford

March J, Wells K (2003), Combining medication and psychotherapy. In: Pediatric Psychopharmacology: Principles and Practice, Martin A, Scahill L, Charney DS, Leckman JF, eds. London: Oxford University Press, pp 426-446

March JS (1995), Cognitive-behavioral psychotherapy for children and adolescents with OCD: a review and recommendations for treatment. $J \mathrm{Am}$ Acad Child Adolesc Psychiatry 34:7-18

McClellan J, Werry J (1997), Practice parameters for the assessment and treatment of children and adolescents with bipolar disorder. J Am Acad Child Adolesc Psychiatry 36:157S-176S

Mendlowitz SL, Manassis K, Bradley S, Scapillato D, Miezitis S, Shaw BF (1999), Cognitive-behavioral group treatments in childhood anxiety disorders: the role of parental involvement. J Am Acad Child Adolesc Psychiatry 38:1223-1229

Menzies RG, Clarke J (1993), A comparison of in vivo and vicarious exposure in the treatment of childhood water phobia. Behav Res Ther 31:9-15

Muris P, Mayer B, Bartelds E, Tierney S, Bogie N (2001), The revised 
version of the Screen for Child Anxiety Related Emotional Disorders (SCARED-R): treatment sensitivity in an early intervention trial for childhood anxiety disorders. Br J Clin Psychol 40:323-336

Muris P, Meesters C, van Melick M (2002), Treatment of childhood anxiety disorders; A preliminary comparison between cognitive-behavioral group therapy and a psychological placebo intervention. $J$ Behav Ther Exp Psychiatry 33:143-158

Nauta MH, Scholing A, Emmelkamp PM, Minderaa RB (2003), Cognitive-behavioral therapy for children with anxiety disorders in a clinical setting: no additional effect of a cognitive parent training. J Am Acad Child Adolesc Psychiatry 42:1270-1278

Orvaschel H, Puig-Antich J (1986), Schedule for Affective Disorders and Schizophrenia for School-Age Children, Epidemiologic Version. Pittsburgh: Western Psychiatric Institute and Clinic

Pina AA, Silverman WK, Fuentes RM, Kurtines WM, Weems CF (2003), Exposure-based cognitive-behavioral treatment for phobic and anxiety disorders: treatment effects and maintenance for Hispanic/Latino relative to European-American youths. J Am Acad Child Adolesc Psychiatry 42:1179-1187

Puig-Antich J, Chambers W (1978), The Schedule for Affective Disorders and Schizophrenia for School-Aged Children. New York: New York State Psychiatric Institute

Rapee R, Spense S, Cobham V, Wignal A (2000), Helping Your Anxious Child: A Step-By-Step Guide for Parents. San Francisco: New Harbinger

Reinecke MA, Dattilio FM, Freeman A (2003), What makes for an effective treatment? In: Cognitive Therapy With Children and Adolescents: A Casebook for Clinical Practice, 2nd ed. Reinecke MA, Dattilio FM, Freeman A, eds. New York: Guilford, pp 1-18

Reinecke MA, Ryan NE, DuBois DL (1998), Cognitive-behavioral therapy of depression and depressive symptoms during adolescence: a review and meta-analysis. J Am Acad Child Adolesc Psychiatry 37:26-34

Rossello J, Bernal G (1999), The efficacy of cognitive-behavioral and interpersonal treatments for depression in Puerto Rican adolescents. J Consult Clin Psychol 67:734-745

Rutter M (1999), The Emanuel Miller Memorial Lecture 1998. Autism: two-way interplay between research and clinical work. J Child Psychol Psychiatry 40:169-188

Sackett D, Richardson W, Rosenberg W, Haynes B (1997), Evidence-Based Medicine. London: Churchill Livingstone

Sackett D, Richardson W, Rosenberg W, Haynes B (2000), Evidence-Based Medicine, 2nd ed. London: Churchill Livingstone

Shadish WR, Robinson L, Congxiao L (1999), ES: A Computer Program for Effect Size Calculation, 1.0 ed. St. Paul, MN: Assessment Systems

Shortt AL, Barrett PM, Fox TL (2001), Evaluating the FRIENDS Program: a cognitive-behavioral group treatment for anxious children and their parents. J Clin Child Psychol 30:525-535
Silverman W, Kurtines W (1996), Anxiety and Phobic Disorders: A Pragmatic Approach. New York: Plenum

Silverman WK (1987), Anxiety Disorders Interview Schedule for Children. Boulder, CO: Graywind Publications

Silverman WK, Albano AM (1996a), The Anxiety Disorders Interview Schedule for DSM-IV: Child Interview Schedule. Boulder, CO: Graywind

Silverman WK, Albano AM (1996b), The Anxiety Disorders Interview Schedule for DSM-IV: Parent Interview Schedule. Boulder, CO: Graywind

Silverman WK, Kurtines WM, Ginsburg GS, Weems CF, Lumpkin PW, Carmichael DH (1999a), Treating anxiety disorders in children with group cognitive-behavioral therapy: a randomized clinical trial. J Consult Clin Psychol 67:995-1003

Silverman WK, Kurtines WM, Ginsburg GS, Weems CF, Rabian B, Serafini LT (1999b), Contingency management, self-control, and education support in the treatment of childhood phobic disorders: a randomized clinical trial. J Consult Clin Psychol 67:675-687

Silverman WK, Nelles WB (1988), The Anxiety Disorders Interview Schedule for Children. J Am Acad Child Adolesc Psychiatry 27:772-778

Spence SH, Donovan C, Brechman-Toussaint M (2000), The treatment of childhood social phobia: the effectiveness of a social skills trainingbased, cognitive-behavioural intervention, with and without parental involvement. J Child Psychol Psychiatry 41:713-726

Thyer BA (1991), Diagnosis and treatment of child and adolescent anxiety disorders. Behav Modif 15:310-325

Treatment for Adolescents with Depression Study (2003), Treatment for Adolescents with Depression Study (TADS): rationale, design, and methods. J Am Acad Child Adolesc Psychiatry 42:531-542

Van Hasselt V, Hersen M (1993), Handbook of Behavior Therapy and Pharmacotherapy for Children. Boston: Longwood

Vostanis P, Feehan C, Grattan E (1998), Two-year outcome of children treated for depression. Eur Child Adolesc Psychiatry 7:12-18

Vostanis P, Feehan C, Grattan E, Bickerton W-L (1996b), Treatment for children and adolescents with depression: lessons from a controlled trial. Clin Child Psychol Psychiatry 1:199-212

Vostanis P, Feehan C, Grattan E, Bickerton W-L (1996a), A randomised controlled out-patient trial of cognitive-behavioural treatment for children and adolescents with depression: 9-month follow-up. J Affect Disord 40:105-116

Weisz JR, Thurber CA, Sweeney L, Proffitt VD, LeGagnoux GL (1997), Brief treatment of mild-to-moderate child depression using primary and secondary control enhancement training. J Consult Clin Psychol 65:703707

Wood A, Harrington R, Moore A (1996), Controlled trial of a brief cognitive-behavioural intervention in adolescent patients with depressive disorders. J Child Psychol Psychiatry 37:737-746 